\title{
USING FLOWS TO CONSTRUCT HILBERT SPACE FACTORS OF FUNCTION SPACES $\left(^{1}\right)$
}

BY

\author{
JAMES KEESLING
}

\begin{abstract}
Let $X$ and $Y$ be metric spaces. Let $G(X)$ be the group of homeomorphisms of $X$ with the compact open topology. The main result of this paper is that if $X$ admits a nontrivial flow, then $G(X)$ is homeomorphic to $G(X) \times l_{2}$ where $l_{2}$ is separable infinite-dimensional Hilbert space. The techniques are applied to other function spaces with the same result. Two such spaces for which our techniques apply are the space of imbeddings of $X$ into $Y, E(X, Y)$, and the space of light open mappings of $X$ into (or onto) $Y, \operatorname{LO}(X, Y)$. Some applications of these results are given. The paper also uses flows to show that if $X$ is the $\sin (1 / x)$-curve, then $G(X)$ is homeomorphic to $l_{2} \times N$, where $N$ is the integers.
\end{abstract}

Introduction. Throughout this paper let $X$ be a metric space and $G(X)$ the group of homeomorphisms of $X$ endowed with the compact open topology. It is well known that under these circumstances $G(X)$ may not be a topological group, although in [4] Arens gives relatively mild conditions on $X$ under which $G(X)$ is a topological group. At present there is an interest in $G(X)$ for $X$ a manifold with boundary. Whittaker has studied the algebraic properties of $G(X)$ for $X$ a compact manifold with boundary and shown that if $G\left(X_{1}\right)$ is algebraically isomorphic to $G\left(X_{2}\right)$, then $X_{1}$ and $X_{2}$ are homeomorphic [30]. Černavskiı [9] and [10], Edwards and Kirby [13], Geoghegan [15], and Mason [23] have studied the topological properties of $G(X)$ for $X$ a manifold. For $X$ a manifold which is not necessarily connected or separable $G(X)$ has the following topological properties:

(1) $G(X)$ is homeomorphic to $G(X) \times l_{2}$ [15]. Actually this is shown for $X$ any locally compact simplicial complex as well in [15].

(2) If $X$ is compact, then $\sigma$-compact subsets of $G(X)$ are negligible, that is, $G(X)$ is homeomorphic to $G(X)-F$ where $F$ is any $\sigma$-compact subset of $G(X)$ [23]. In [15] this is shown to be true of $G(X)$ for $X$ any separable manifold or any separable locally compact simplicial complex.

Received by the editors September 2, 1970 and, in revised form, January 15, 1971.

AMS 1970 subject classifications. Primary 57E05, 54C10, 54C35; Secondary 54F45, 54H15, 57A20, 54B20.

Key words and phrases. Flow, group of homeomorphisms, space of imbeddings, space of light mappings, infinite-dimensional manifold, Hilbert space, Hilbert cube, hyperspace, negligible subsets.

${ }^{(1)}$ This research was entirely supported by a Summer Research Grant awarded the author by the Graduate School of the University of Florida during the summer of 1970.

Copyright (C) 1971, American Mathematical Society 
(3) For a large class of manifolds, $X$ including the compact ones with boundary $G(X)$ is locally contractible [9] and [10], [13], and [22]. It should be remarked that if $X$ is the plane $R^{2}$ less an infinite closed discrete set, then $G(X)$ is not locally contractible. Thus $G(X)$ is not always locally contractible even if $X$ is a connected manifold without boundary. What one would like to show is that $G(X)$ is a separable Fréchet manifold whenever $X$ is a compact manifold [3]. (By a separable Fréchet manifold is meant a separable metric space which is locally homeomorphic to $l_{2}$ where $l_{2}$ is a separable infinite-dimensional Hilbert space. These are called topological Banach manifolds in [3] and are required to be connected there.) There are several proofs that $G(X)$ is a separable Fréchet manifold when $X$ is a 1-dimensional manifold (the first proof was by Anderson [2]), but for higher dimensional manifolds the problem remains unsolved.

If $X$ is the Hilbert cube, it is known that $G(X)$ has the three properties enumerated above also (see [28], [31], and [15]). Actually $G(X)$ is contractible in this case [28] and the conjecture is that it is homeomorphic to $l_{2}$ [3]. It would be equivalent to prove in this case that $G(X)$ is a separable Fréchet manifold by [17]. Some topological properties of $G(X)$ for other metric spaces have been studied by Brechner [7] and [8] and Keesling [21].

The main result of this paper is that for a large class of metric spaces $X, G(X)$ is homeomorphic to $G(X) \times l_{2}$. Specifically if $X$ admits a nontrivial flow (a flow is a topological group action of the additive reals on $X$ ), then $G(X)$ has $l_{2}$ as a topological factor. As pointed out in [15] a result in [1] then implies that $\sigma$-compact subsets of $G(X)$ are negligible if $G(X)$ is separable metric. Therefore if $X$ is locally compact separable metric and admits a nontrivial flow, then $\sigma$-compact subsets of $G(X)$ are negligible. These results are proved in $\S 3$ of the paper. Actually our results apply to other function spaces as well as $G(X)$ as we point out in $\S 4$. We believe that our techniques will have other applications as well. It is significant that our results hold even though the function spaces involved may have very bad separation properties. They may not even be normal, let alone metrizable. Also in the case of $G(X)$, the results hold even when $G(X)$ is not a topological group.

Flows on spaces occur in very general circumstances and so we consider our results a substantial improvement of the results in [15] and [23]. One can easily show that all spaces admitting cone patches [15] and all manifolds [23] admit nontrivial flows and thus the results in this paper are a proper generalization. However, an important part of our proof is from Geoghegan's extension of Morse's results [15]. These results are summarized for our purposes in $\$ 2$ of this paper. Besides other spaces the results here apply to the homeomorphism group of the hyperspace of a space admitting a flow. Using flows we also construct homeomorphisms of the hyperspace which are the identity on the singleton sets.

In $\$ 6$ of the paper we use flows to show that the group of homeomorphisms of the familiar $\sin (1 / x)$-curve is homeomorphic to $l_{2} \times N$ where $N$ is the integers. There are other related results in this section as well. 
Notation. As has already been indicated $l_{2}$ denotes separable infinitedimensional Hilbert space as a topological space. If $Z$ and $Q$ are topological spaces, then $Z \sim Q$ will mean that $Z$ is homeomorphic to $Q$.

It would be helpful if the reader had some previous knowledge of function spaces and the compact open topology. If $X$ and $Y$ are metric spaces, we denote the continuous functions from $X$ to $Y$ by $C(X, Y)$ and we give this set the compact open topology. If $K$ is compact in $X$ and $V$ is open in $Y$, then

$$
(K, V)=\{f \in C(X, Y): f[K] \subset V\} .
$$

The compact open topology has as a subbasis all sets of the form $(K, V)$. If $F(X, Y)$ is a subspace of $C(X, Y)$, then $(K, V)$ will denote $(K, V) \cap F(X, Y)$. Context will make clear what is intended by $(K, V)$ and this convention will reduce our notation considerably. A useful reference which deals with the compact open topology is Chapter 12 of [12]. We also want to observe here that we will be assuming that both $X$ and $Y$ are metric spaces and thus $k$-spaces [12, 9.3, p. 248]. Thus we will have available all of the special theorems on function spaces where the spaces involved are $k$-spaces in Chapter 12 of [12]. For instance we have the theorem $C(X, C(Y, Z)) \sim C(X \times Y, Z)$ provided $X \times Y$ is a $k$-space [12, Theorem 5.3, p. 265]. The reader should observe the caveat that if $X \times Y$ is not a $k$-space, then $C(X, C(Y, Z))$ may not be homeomorphic to $C(X \times Y, Z)$.

1. The dimension of $G(X)$. Recall that we are assuming that $X$ is a metric space throughout the paper. In [21] it is shown that if $G(X)$ is locally compact, then $\operatorname{dim} G(X)=0$. In [7] it is shown that there is a compact metric space $X$ with the property that $\operatorname{dim} G(X)=1$. In [8] it is shown that if $X$ admits a nontrivial flow then $\operatorname{dim} G(X)=\infty$. This result is also a corollary to Theorem III. 2 of this paper. Using a theorem of Gleason we are able to improve this result.

I.1. THEOREM If $X$ is compact or locally compact and locally connected and $G(X)$ contains an arc, then $\operatorname{dim} G(X)=\infty$.

Proof. Suppose that $A$ is an arc in $G(X)$. By the results in [4] $G(X)$ is a topological group. We may suppose that $e \in A$ where $e$ is the identity homeomorphism of $X$. Let $B=\bigcup_{n=1}^{\infty}\left(A \cup A^{-1}\right)^{n}$. Then $B$ is a subgroup of $G(X)$ and $B$ is arcwise connected. We have two cases.

Case (i). $\operatorname{dim} B=\infty$.

In this case we note that $B$ is $\sigma$-compact and thus Lindelöf. Since $G(X)$ is regular, so is $B$ and thus $B$ is normal. Let $k$ be a positive integer. By [27, Theorem VII.2, p. 193], there is a compact set $K$ in $B$ with $\operatorname{dim} K \geqq k$. But then $\operatorname{dim} G(X) \geqq k$ also. Therefore $\operatorname{dim} G(X)=\infty$ in this case.

Case (ii). $\operatorname{dim} B<\infty$.

In this case let $B^{*}$ be the locally arcwise connected group associated with $B$ [16, Definition 3.3]. By Corollary 7.3 of [16] $B^{*}$ is a Lie group and thus contains a nontrivial one parameter subgroup. This implies that $B$ contains a nontrivial one 
parameter subgroup as well [16, Theorem 3.2(5)]. Since $X$ is a $k$-space, this implies that $X$ admits a nontrivial flow [12, Corollary 3.2, p. 261]. Now apply the results of [8] or Theorem III.2 of this paper to say that $\operatorname{dim} G(X)=\infty$. The proof of the theorem is now complete.

I.2. RemARK. If $G$ is any topological group which contains an arc we have essentially shown that either $\operatorname{dim} G=\infty$ or $G$ contains a nontrivial one parameter subgroup.

I.3. EXAMPLE. Let $\mathscr{B}$ be the Lebesgue measurable subsets of $[0,1]$ and let Lebesgue measure be denoted by $\lambda$. Let $\mathscr{N}$ be the sets of Lebesgue measure zero. The operation of symmetric difference on $\mathscr{B}, A \triangle B=(A-B) \cup(B-A)$, is a group operation and $\mathscr{N}$ is a subgroup of $\mathscr{B}$. Let $S(\lambda)=\mathscr{B} / \mathscr{N}$. Define the metric $d$ on $S(\lambda)$ by $d(\{A\},\{B\})=\lambda(A \triangle B)$ for $A, B \in \mathscr{B}$. Then $S(\lambda)$ is a topological group in this topology. The mapping $C:[0,1] \times S(\lambda) \rightarrow S(\lambda)$ defined by $C(t,\{A\})=\{t A\}$ $=\{\{t a: a \in A\}\}$ can be easily seen to be a contraction of $S(\lambda)$ to a point. Thus $S(\lambda)$ contains many arcs, but no one parameter subgroups since every element of $S(\lambda)$ has order two. One can easily verify that $S(\lambda)$ is infinite dimensional as Remark I.2 implies. Bessaga and Pelczyński have recently shown that $S(\lambda)$ is actually homeomorphic to $l_{2}[6]$.

2. Parameterizing curves. In this section we give a summary of the results from Morse [25] and their extension in [15] which will be needed in the next section. No proofs are given since we are simply restating results in $\S 1$ of [15] to have them in convenient form for reference. One should consult $\S 1$ of [15] for Geoghegan's proofs of these results.

II.1. Definition. Let $I$ be the unit interval $[0,1]$ and let $C(I, X)$ be the continuous functions from $I$ into $X$. Then let

$$
\begin{aligned}
C^{\prime}(I, X) & =\{f \in C(I, X): f \text { is not constant }\}, \\
D(I, X) & =\{f \in C(I, X): f \text { is not constant on any interval }\}, \\
E(I, X) & =\{f \in C(I, X): f \text { is an imbedding }\}, \\
M_{0}(I) & =\{f \in C(I, I): f \text { is monotone increasing and onto }\}, \\
H_{0}(I) & =\left\{f \in M_{0}(I): f \text { is a homeomorphism }\right\} .
\end{aligned}
$$

We endow these sets of functions with the compact open topology, which in this case is the same as that induced by the metric $d(f, g)=\sup \{d(f(x), g(x)): x \in I\}$.

II.2. Definition. Let $n \geqq 2$ be an integer and let

$$
A_{n}=\left\{\left(t_{1}, \ldots, t_{n}\right): 0 \leqq t_{1} \leqq \cdots \leqq t_{n} \leqq 1\right\} .
$$

Let $f \in C(I, X)$ and define $\delta\left(f, t_{1}, \ldots, t_{n}\right)=\min \left\{d\left(f\left(t_{i}\right), f\left(t_{i+1}\right)\right): 1 \leqq i \leqq n-1\right\}$. Let $\mu_{n}(f)=\sup \left\{\delta\left(f, t_{1}, \ldots, t_{n}\right):\left(t_{1}, \ldots, t_{n}\right) \in A_{n}\right\}$. Then let

$$
\mu(f)=\sum_{n=2}^{\infty} 2^{1-n} \mu_{n}(f) .
$$

It is shown in [15] and [25] that $\mu$ is continuous from $C(I, X)$ into $[0, \infty)$. Now let $k_{t}: I \rightarrow I$ be defined by $k_{t}(s)=t s$ for $0 \leqq t \leqq 1$. Let $f \in C(I, X)$ and define $f_{t}$ 
$=f \circ k_{t}$. Then $\mu\left(f_{t}\right)$ ranges between 0 and $\mu(f)$. If $0 \leqq s \leqq \mu(f)$, then there is a $t_{s}$ such that $\mu\left(f_{t_{s}}\right)=s$. If $f_{\mu}: I \rightarrow X$ is defined by $f_{\mu}(s / \mu(f))=f\left(t_{s}\right)$ for $0 \leqq s \leqq \mu(f)$, then $f_{\mu}$ is well defined and continuous for all $f \in C^{\prime}(I, X)$. Also $f_{\mu}\left(\mu\left(f_{t}\right) / \mu(f)\right)=f(t)$. For the constant functions in $C(I, X)$ let $f_{\mu}=f$.

II.3. Proposition. Let $\psi: C(I, X) \rightarrow C(I, X)$ be defined by $\psi(f)=f_{\mu}$. Then $\psi$ is a continuous retraction and the image of $C^{\prime}(I, X)$ under $\psi$ is contained in $D(I, X)$. Furthermore, the image of $E(I, X)$ is contained in $E(I, X)$. If $f \in C(I, X)$, then $\mu(f)$ has the same range as $f$ does.

We denote the image of $E(I, X)$ under $\psi$ as $E_{\mu}(I, X)$ and the image of $D(I, X)$ as $D_{\mu}(I, X)$.

II.4. Definition. Now let $r: C^{\prime}(I, X) \rightarrow M_{0}(I)$ be defined by $r(f)(t)=\mu\left(f_{t}\right) / \mu(f)$. Then $\psi(f) \circ r(f)=f$ as pointed out above.

II.5. Proposition. The map $r: C^{\prime}(I, X) \rightarrow M_{0}(I)$ is continuous. For $f \in D(I, X)$, $r(f) \in H_{0}(I)$. The map $G: C^{\prime}(I, X) \rightarrow D_{\mu}(I, X) \times M_{0}(I)$ defined by $G(f)=(\psi(f), r(f))$ is a homeomorphism of $C^{\prime}(I, X)$ onto $D_{\mu}(I, X) \times M_{0}(I)$. Also $G$ restricted to $E(I, X)$ maps onto $E_{\mu}(I, X) \times H_{0}(I)$ and $G$ restricted to $D(I, X)$ maps onto $D_{\mu}(I, X) \times H_{0}(I)$.

The continuity of $G$ in Proposition II.5 is proved in Theorem 1.17 of [15]. We will need Propositions II.3 and II.5 in the sequel.

3. Factors of $G(X)$. In this section we show that any metric space which admits a nontrivial flow has the property that $G(X) \sim G(X) \times l_{2}$. Certain other function spaces also have this property. These further results are stated and their proofs indicated at the end of this section and in the next section. We prove Theorem III. 2 in detail. We scurry over the details in the proofs of the other related theorems since they are similar to the details in the proof of Theorem III.2.

An essential part of the proof of Theorem III.2 depends on the fact that $H_{0}(I)$ $\sim l_{2}$. There are at least two proofs of this theorem which are currently circulating. There is a manuscript of R. D. Anderson [2] containing one proof discovered by him using results in [1]. The following proof was communicated to me by James West who heard it from R. Connelly who heard it from Morton Brown.

III.1. THEOREM. $H_{0}(I) \sim l_{2}$.

Proof. We will only sketch the proof. The details are routine to verify. We will show that $H_{0}(I)$ is homeomorphic to $\prod_{n=0}^{\infty} \prod_{i=1}^{2^{n}}(0,1)_{n, i}$. It will then follow that $H_{0}(I)$ is homeomorphic to $l_{2}$ by [3]. Let $\left(x_{n, i}\right) \in \prod_{n=0}^{\infty} \prod_{i=1}^{2^{n}}(0,1)_{n, i}$. We will define an orientation preserving homeomorphism $h$ of $I$ associated with $\left(x_{n, i}\right)$. Suppose that $n$ is given and that we have defined points $A_{n}=\left\{0=\alpha_{0}^{n}<\alpha_{1}^{n}<\cdots<\alpha_{2}^{n}=1\right\}$ and $B_{n}=\left\{0=\beta_{0}^{n}<\beta_{1}^{n}<\cdots<\beta_{2}^{n}=1\right\}$ and defined $h$ such that $h\left(\alpha_{i}^{n}\right)=\beta_{i}^{n}$ for $i=0,1, \ldots, 2^{n}$. We extend the definition of $h$ to a set of points $A_{n+1} \supset A_{n}$ onto $B_{n+1} \supset B_{n}$ with each of $A_{n+1}$ and $B_{n+1}$ having $2^{n+1}+1$ points. If $n$ is odd, then let $z_{i}$ be the midpoint of 
the interval $\left[\alpha_{i-1}^{n}, \alpha_{i}^{n}\right]$ for $i=1,2, \ldots, 2^{n}$ and let $y_{i}=h\left(z_{i}\right)=x_{n, i}\left(\beta_{i}^{n}-\beta_{i-1}^{n}\right)+\beta_{i-1}^{n}$. If $n$ is even, then let $y_{i}$ be the midpoint of the interval $\left[\beta_{i-1}^{n}, \beta_{i}^{n}\right]$ and let $z_{i}=h^{-1}\left(y_{i}\right)$ $=x_{n, i}\left(\alpha_{i}^{n}-\alpha_{i-1}^{n}\right)+\alpha_{i-1}^{n}$. Then let $A_{n+1}=A_{n} \cup\left\{z_{i}\right\}_{i=1}^{2^{n}}$ and $B_{n+1}=B_{n} \cup\left\{y_{i}\right\}_{i=1}^{2^{n}}$. Then we will have $A_{n+1}=\left\{0=\alpha_{0}^{n+1}<\cdots<\alpha_{2^{n+1}}^{n+1}=1\right\}$ and $B_{n+1}=\left\{0=\beta_{0}^{n+1}<\cdots<\beta_{2^{n+1}}^{n+1}=1\right\}$ with $h\left(\alpha_{i}^{n+1}\right)=\beta_{i}^{n+1}$. Proceeding in this way and letting $A=\bigcup_{n=1}^{\infty} A_{n}$ and $B$ $=\bigcup_{n=1}^{\infty} B_{n}, A$ and $B$ will be dense in $I$ and $h[A]=B$ will be order preserving. Thus $h$ will have a continuous extension to an orientation preserving homeomorphism of $I$ onto itself which will be denoted by $h$ also. If we let $F: \prod_{n=0}^{\infty} \prod_{i=1}^{2^{n}}(0,1)_{n, i}$ $\rightarrow H_{0}(I)$ be defined by $F\left(\left(x_{n, i}\right)\right)=h$, then $F$ is the desired homeomorphism of $\prod_{n=0}^{\infty} \prod_{i=1}^{2^{n}}(0,1)_{n, i}$ onto $H_{0}(I)$.

III.2. TheOrem. Suppose that $X$ admits a nontrivial flow. Then $G(X) \sim G(X) \times l_{2}$.

Proof. Let $F: R \times X \rightarrow X$ be a nontrivial flow on $X$ and let $x_{0}$ have a nondegenerate orbit under $F$. Let $t_{0}$ be a positive real number such that $F\left(t_{0}, x_{0}\right) \neq x_{0}$. Let $U_{1}$ and $U_{2}$ be disjoint open subsets of $X$ such that $x_{0} \in U_{1}$ and $F\left(t_{0}, x_{0}\right) \in U_{2}$. Then let $V \subset U_{1}$ be an open neighborhood of $x_{0}$ such that for all $y \in V, F\left(t_{0}, y\right) \in U_{2}$. Now if $y \in V$, then if $A_{y}$ is the orbit of $y$ under $F$ and $B_{y}$ is the arc component of $A_{y} \cap V$, then $B_{y}$ is clearly homeomorphic to $R$ and $\bar{B}_{y}$ is an arc in $X$. By Beck's result in [5] as restated in Theorem 2 of [21], there is a flow $F^{\prime}$ on $X$ whose set of fixed points is $X-V$ and whose orbits are the sets $\left\{B_{y}: y \in V\right\} \cup\{\{x\}: x \in X-V\}$. On p. 270 of [29] Whitney gives an elegant proof of the existence of a local crosssection for the flow $F^{\prime}$ at the point $x_{0}$. Call this local cross-section $N$. Then $N$ has the property that (1) $N$ is closed; (2) there is a neighborhood $O$ of $x_{0}$ such that every orbit of $F^{\prime}$ meeting $O$ also meets $N$; and (3) $N$ has at most one point from each orbit of $F^{\prime}$. We will have no further use for the original flow $F$, so let us denote $F^{\prime}$ by $F$.

Claim 1. The set $F[[-1,1] \times N]$ is closed in $X$ and homeomorphic to $[-1,1]$ $\times N$ under the mapping $F$.

Proof of Claim 1. Suppose that $F\left(t_{i}, x_{i}\right) \rightarrow z$ with $x_{i} \in N$ and $t_{i} \in[-1,1]$ for all $i$. Then there is a subsequence $\left\{t_{i}\right\}$ converging to $t_{0}$ for some $t_{0} \in[-1,1]$. Resubscript and call this subsequence $\left\{t_{i}\right\}$. Then $F\left(t_{i}, x_{i}\right) \rightarrow z$ still. Since $F$ is a flow, $F\left(t_{i}-t_{0}, x_{i}\right) \rightarrow F\left(-t_{0}, z\right)$. But this implies that $x_{i} \rightarrow F\left(-t_{0}, z\right)$. Therefore $F\left(-t_{0}, z\right) \in N$ since each $x_{i} \in N$. But then $z=F\left(t_{0}, F\left(-t_{0}, z\right)\right)$ and $z \in F[[-1,1] \times N]$ and we have shown that $F[[-1,1] \times N]$ is closed in $X$. Now to show that the restriction of $F$ to $[-1,1] \times N$ is an imbedding. Clearly $F \mid[-1,1] \times N$ is one-to-one and continuous. Now suppose that $z_{i}=F\left(t_{i}, x_{i}\right) \rightarrow z_{0}=F(t, x)$ and suppose that $\left(t_{i}, x_{i}\right) \rightarrow(t, x)$ in $[-1,1] \times N$. There are two cases. First of all, suppose that $x_{i} \rightarrow x$ in $N$. Then $t_{i} \rightarrow t$. By taking a subsequence of $\left\{t_{i}\right\}$ and resubscripting we may suppose that $t_{i} \rightarrow p \neq t$ in $[-1,1]$. Then $F\left(t_{i}, x_{i}\right) \rightarrow F(p, x)$. However, $F(p, x) \neq F(t, x)$ since $F \mid[-1,1] \times N$ is one-to-one. But now we have a sequence converging to two distinct points in $X$, a contradiction. In the second case suppose that $x_{i} \rightarrow x$. Then there is a subsequence of $\left\{x_{i}\right\}$ such that no subsequence of it converges to $x$. Call 
that subsequence $\left\{x_{i}\right\}$ by resubscripting. We may suppose that $t_{i} \rightarrow p$ for some $p \in[-1,1]$ by resubscripting if necessary. Now $F\left(t_{i}, x_{i}\right) \rightarrow F(t, x)$ still. Since $t_{i} \rightarrow p$ we then have also that $F\left(t_{i}-p, x_{i}\right) \rightarrow F(t-p, x)$. Of course, $t_{i}-p \rightarrow 0$ and thus $x_{i} \rightarrow F(t-p, x)$ which places $F(t-p, x)$ in $N$. Therefore $F\left(t_{i}, x_{i}\right)$ $\rightarrow F(p, F(t-p, x))=F(t, x)$. Since $F[-1,1] \times N$ is one-to-one, $p=t$ and $x$ $=F(t-p, x)$. But this implies that $x_{i} \rightarrow x$, a contradiction. We have now shown that if $F\left(t_{i}, x_{i}\right) \rightarrow F(t, x)$, then $\left(t_{i}, x_{i}\right) \rightarrow(t, x)$ and Claim 1 is now proved.

We now return to the proof of Theorem III.2. Let $Q \subset N$ be the set of points in $N$ which are interior to $F[[-1,1] \times N]$ in $X$. Then at least the point $x_{0}$ is in $Q$. Now let $f: N \rightarrow[0,1]$ be a continuous function having the property that $f\left(x_{0}\right)=1$ and $f^{-1}(0)=N-Q$. Now let $Z=\{(t, x) \in[-1,1] \times N:|t| \leqq f(x)\}$.

Claim 2. The set $F[Z]$ is closed in $X$, int ${ }_{X} F[Z]=\{F(t, x): x \in Q$ and $|t|<f(x)\}$, and $\operatorname{int}_{X} F[[-1,1] \times N]=\{F(t, x):|t|<1$ and $x \in Q\}$.

Proof of Claim 2. Since $F[[-1,1] \times N]$ is closed in $X$ and $Z$ is closed in $[-1,1] \times N$ and $F \mid[-1,1] \times N$ is an imbedding we immediately have that $F[Z]$ is closed in $X$. If we can show that int $_{X} F[[-1,1] \times N]$ is the set in the claim, then int $_{X} F[Z]$ can easily be seen to be the set claimed by using the fact that $F \mid[-1,1]$ $\times N$ is an imbedding. So we will consider the claim proved when we have shown that int $_{X} F[[-1,1] \times N]$ is the set claimed. Let $z=F(t, x) \in$ int $_{X} F[[-1,1] \times N]$. If $t=1$, then let $\varepsilon_{i} \rightarrow 0$ with $\varepsilon_{i}>0$ for all $i$. Then $z_{i}=F\left(t+\varepsilon_{i}, x\right)$ converges to $z$ and $z_{i} \notin F[[-1,1] \times N]$ for all $i$. Therefore $z \notin$ int $_{X} F[[-1,1] \times N]$, a contradiction. Therefore $t \neq 1$. Similarly $t \neq-1$ and thus $|t|<1$. Now suppose that $x \notin Q$. Then there is a sequence $x_{i} \rightarrow x$ with $x_{i} \notin F[[-1,1] \times N]$ for all $i$. But then $F\left(t, x_{i}\right) \rightarrow F(t, x)=z$. Since $z \in$ int $_{X} F[[-1,1] \times N]$, this sequence is finally in $F[[-1,1] \times N]$. Thus $F\left(t, x_{i}\right)=F\left(t_{i}, y_{i}\right)$ with $\left(t_{i}, y_{i}\right) \in[-1,1] \times N$ for all $i \geqq K$ for some integer $K$. Since $F \mid[-1,1] \times N$ is an imbedding we must have that $\left(t_{i}, y_{i}\right)$ $\rightarrow(t, x)$ and thus $t_{i}-t \rightarrow 0$. But $F\left(t_{i}-t, y_{i}\right)=x_{i}$. Let $\left|t_{i}-t\right|<1$. Then for that $i$, $x_{i} \in F[[-1,1] \times N]$, a contradiction. Therefore $x \in Q$ and we have shown that int $_{X} F[[-1,1] \times N] \subset\{F(t, x):|t|<1, x \in Q\}$. Now suppose that $z=F(t, x)$ with $|t|<1$ and $x \in Q$ and suppose that $z_{i} \rightarrow z$ with $z_{i} \notin F[[-1,1] \times N]$ for all $i$. Then $F\left(-t, z_{i}\right) \rightarrow F(-t, z)=x$. Since $x \in Q, F\left(-t, z_{i}\right)$ is finally in $F[[-1,1] \times N]$. Therefore $F\left(-t, z_{i}\right)=F\left(t_{i}, x_{i}\right)$ with $\left(t_{i}, x_{i}\right) \in[-1,1] \times N$ for some residual subsequence of $\left\{F\left(-t, z_{i}\right)\right\}$. Again using the fact that $F \mid[-1,1] \times N$ is an imbedding we have that $\left(t_{i}, x_{i}\right) \rightarrow(0, x)$. Thus $t_{i} \rightarrow 0$. But then let $K$ be such that for $i \geqq K$, $\left|t+t_{i}\right|<1$. Then for $i \geqq K$ we have $z_{i}=F\left(t+t_{i}, x_{i}\right)$ and $z_{i} \in F[[-1,1] \times N]$, a contradiction. That is, $z \in$ int $_{X} F[[-1,1] \times N]$, and we have now shown that int $_{X} F[[-1,1] \times N]=\{F(t, x):|t|<1$ and $x \in Q\}$ and Claim 2 is now proved.

Returning to the proof of Theorem III. 2 we now want to describe a way of factoring the group of homeomorphisms of $X$. We want to show that $G(X)$ is homeomorphic to $G_{\text {can }}(X) \times F H(X)$ where $G_{\text {can }}(X)$ and $F H(X)$ will be defined shortly. We will then show that $F H(X)$ is homeomorphic to $F H(X) \times l_{2}$. We will then obviously have proved that $G(X) \sim G(X) \times l_{2}$. 
Now let

$$
\begin{aligned}
& F H(X)=\left\{h \in G(X): h \mid X-\text { int }_{X} F[Z]\right. \text { is the identity and } \\
& \qquad h[F[[-f(x), f(x)] \times\{x\}]]=F[[-f(x), f(x)] \times\{x\}] \text { for all } x \in N\} .
\end{aligned}
$$

If $h \in F H(X)$, then $h$ will be called a fiber homeomorphism. For $x \in Q \subset N$ the arc $F[[-f(x), f(x)] \times\{x\}]$ will be called the fiber through $x$. Let $x \in Q \subset N$ and let $g_{\varepsilon}:[0,1] \rightarrow[-\varepsilon, \varepsilon]$ be a linear map with $g_{\varepsilon}(0)=-\varepsilon$ and $g_{\varepsilon}(1)=\varepsilon$. Then let $g_{x}:[0,1] \rightarrow[-1,1] \times N$ be defined by $g_{x}(t)=\left(g_{f(x)}(t), x\right)$. Then the image of $[0,1]$ under the map $F \circ g_{x}$ is just the fiber through $x$ in $X$. Now we can define $G_{\text {can }}(X)$ $=\left\{h \in G(X):\right.$ for all $\left.x \in Q, h \circ F \circ g_{x}=\psi\left(h \circ F \circ g_{x}\right)\right\}$ where $\psi$ is defined in $\S 2$ of the paper. This could also have been expressed as $h \circ F \circ g_{x}=\left(h \circ F \circ g_{x}\right)_{\mu}$ as in [15] and [25].

Claim 3. $G(X) \sim G_{\text {can }}(X) \times F H(X)$.

Proof of Claim 3. Let $h \in G(X)$. We will define mappings $J: G(X) \rightarrow G_{\text {can }}(X)$ and $K: G(X) \rightarrow F H(X)$ such that $h=J(h) \circ K(h)$. We will show that $J$ and $K$ are continuous. We will then show that if $P: G(X) \rightarrow G_{\text {can }}(X) \times F H(X)$ is defined by $P(h)=(J(h), K(h))$, then $P$ is a homeomorphism.

We now define $J: G(X) \rightarrow G_{\text {can }}(X)$. Let $h \in G(X)$ and let $J(h)(x)=h(x)$ for $x \in X-$ int $_{X} F[Z]$. For $x \in Q$ let $J(h)$ be defined on the fiber through $x$ so that $J(h) \circ F \circ g_{x}=\psi\left(h \circ F \circ g_{x}\right)$. At this point it should be clear that: (1) $J(h)$ is a welldefined function from $X$ onto $X$ which is one-to-one; (2) $J(J(h))=J(h)$ since $\psi$ is a retraction (Proposition II.3); and (3) if $J(h)$ is a homeomorphism of $X$, then $J(h) \in G_{\text {can }}(X)$ since $\psi$ is a retraction. Thus we will only have to show that $J(h)$ is a homeomorphism of $X$ and that $J$ is continuous. Now to show that $J(h)$ is a homeomorphism it will only be necessary to show that $J(h) \mid F[Z]$ is an imbedding since $J(h)$ agrees with $h$ on $X-$ int $_{X} F[Z]$ and $J(h)[F[Z]]$ is closed in $X$. We will show that $J(h) \circ F: Z \rightarrow X$ is an imbedding. It will then readily follow that $J(h) \mid F[Z]$ is an imbedding also. Now suppose that $\left(t_{i}, x_{i}\right) \rightarrow(t, x)$ in $Z \subset[-1,1]$ $\times N$. If $x \in N-Q$, then $t_{i} \rightarrow 0$ and clearly $J(h) \circ F\left(t_{i}, x_{i}\right) \rightarrow J(h) \circ F(t, x)$ $=h \circ F(t, x)$. Now if $x \in Q$, then we may suppose that $x_{i} \in Q$ for all $i$ since $Q$ is open in $N$. But then $\left(\bigcup_{i=1}^{\infty}\left[-f\left(x_{i}\right), f\left(x_{i}\right)\right] \times\left\{x_{i}\right\}\right) \cup[-f(x), f(x)] \times\{x\}$ is homeomorphic to $[0,1] \times\left(\left\{x_{i}\right\}_{i=1}^{\infty} \cup\{x\}\right)$. But then $J(h) \circ F$ is continuous on this set by the continuity of $\psi$ in Proposition II.3 and Theorem 3.1, p. 261 of [12]. Thus we have that $J(h) \circ F\left(t_{i}, x_{i}\right) \rightarrow J(h) \circ F(t, x)$. Therefore $J(h) \circ F: Z \rightarrow X$ is continuous. Now suppose that $J(h) \circ F\left(t_{i}, x_{i}\right) \rightarrow J(h) \circ F(t, x)$. Then clearly $x_{i} \rightarrow x$. Now if $x \in N-Q$, then $t_{i} \rightarrow 0$ and $t=0$. Thus $t_{i} \rightarrow t$ in this case. If $x \in Q$, then we may suppose that $x_{i} \in Q$ for all $i$. Now $J(h) \circ F$ restricted to

$$
\left(\bigcup_{i=1}^{\infty}\left[-f\left(x_{i}\right), f\left(x_{i}\right)\right] \times\left\{x_{i}\right\}\right) \cup[-f(x), f(x)] \times\{x\}
$$

is an imbedding since this set is compact and $J(h) \circ F$ is continuous and one-to-one. Therefore $\left(t_{i}, x_{i}\right) \rightarrow(t, x)$ in this case also. Therefore $J(h) \circ F \mid Z$ is an imbedding and $J(h)$ is a homeomorphism as asserted. 
We will now show that $J$ is continuous from $G(X)$ to $G_{\text {can }}(X)$. Let $h \in G(X)$ and $J(h) \in(K, V)$ where $K$ is compact in $X$ and $V$ is open in $X$. This notation was discussed in the Notation Section. Let $K^{\prime}=\{F(t, x):|t| \leqq f(x)$ with $x \in N$ where $F(q, x) \in K$ for some $|q| \leqq f(x)\} \cup K$. Now suppose that for all $\varepsilon>0$, there is an $h_{\varepsilon}$ such that $d\left(h_{\varepsilon}(x), h(x)\right)<\varepsilon$ for all $x \in K^{\prime}$ and $J\left(h_{\varepsilon}\right) \notin(K, V)$. Then we can construct a sequence $x_{i} \rightarrow x$ in $K$ and $h_{i} \in G(X)$ such that $h_{i}$ converges uniformly on $K^{\prime}$ to $h$ and such that $J\left(h_{i}\right)\left(x_{i}\right) \notin V$ for all $i$. Now if $x_{i,} \notin$ int $_{X} F[Z]$ for some subsequence of $\left\{x_{i}\right\}$, then $x_{i} \rightarrow x$ and since $J\left(h_{i}\right) \equiv h_{i}$ and $J(h) \equiv h$ on $X$-int $x_{X} F[Z]$, we would have $J\left(h_{i}\right)\left(x_{i,}\right)=h_{i,}\left(x_{i,}\right) \rightarrow h(x)=J(h)(x)$ since $h_{i}$ converges to $h$ uniformly on $K^{\prime}$. But then $J\left(h_{i}\right)\left(x_{i j}\right)$ would have to be finally in $V$, a contradiction. Therefore $\left\{x_{i}\right\}$ has to be finally in $\operatorname{int}_{X} F[Z]$. Now suppose that $x \in N-Q$. Then let $x_{i}$ $=F\left(t_{i}, y_{i}\right)$ where $\left(t_{i}, y_{i}\right) \in Z \subset[-1,1] \times N$. Then $y_{i} \rightarrow x$ and if we let $G_{i}$ be the fiber through $y_{i}$ in $X$, then $G_{i} \rightarrow x$. Since $h_{i}$ converges uniformly to $h$ on $K^{\prime}$ we have that $h_{i}\left[G_{i}\right] \rightarrow h(x)$. However, $h_{i}\left[G_{i}\right]=J\left(h_{i}\right)\left[G_{i}\right]$ and $J\left(h_{i}\right)\left(x_{i}\right) \in J\left(h_{i}\right)\left[G_{i}\right]$. Thus $J\left(h_{i}\right)\left(x_{i}\right) \rightarrow h(x)=J(h)(x)$, contradicting the fact that $J\left(h_{i}\right) \notin V$ for all $i$. Therefore we are left with the alternative that $x=F(q, y)$ for some $(q, y) \in Z$ with $y \in Q$. Then let $x_{i}=F\left(t_{i}, y_{i}\right)$ with $\left(t_{i}, y_{i}\right) \in Z$. Then $\left(t_{i}, y_{i}\right) \rightarrow(q, y)$. Now $h_{i} \circ F \circ g_{y_{i}}$ converges uniformly to $h \circ F \circ g_{y}$ by the continuity of composition (Theorem 2.2, p. 259, [12]) and by the continuity of $\psi$ we have that $\psi\left(h_{i} \circ F \circ g_{y_{i}}\right)$ converges uniformly to $\psi\left(h \circ F \circ g_{y}\right)$. But this implies that $J\left(h_{i}\right) \circ F \circ g_{y_{i}}\left(t_{i}, y_{i}\right) \rightarrow J(h) \circ F \circ g_{y}(q, y)$. This contradicts the fact that $J\left(h_{i}\right)\left(x_{i}\right) \notin V$ for all $i$. Therefore we have finally shown that there is an $\varepsilon>0$ such that if $d(h(x), g(x))<\varepsilon$ for all $x \in K^{\prime}$ with $g \in G(X)$, then $J(g) \in(K, V)$. This implies that $J$ is continuous since $(K, V)$ was an arbitrary subbasic element.

We now define $K: G(X) \rightarrow F H(X)$. Let $h \in G(X)$. Then let $K(h) \mid X-$ int $_{X} F[Z]$ be the identity and for $x \in Q$ let $K(h)$ take the fiber through $x$ onto itself such that $g_{x}^{-1} \circ F^{-1} \circ K(h) \circ F \circ g_{x}:[0,1] \rightarrow[0,1]$ is just $r\left(h \circ F \circ g_{x}\right)$. Then by II.4 and II. 5 we easily have $J(h) \circ K(h)=h$. This implies that $K(h)=J(h)^{-1} \circ h$ and therefore $K(h)$ is a homeomorphism. It should also be clear that $K(h)$ is also a fiber homeomorphism. To show that $K$ is continuous from $G(X)$ to $F H(X)$ involves an argument similar to showing that $J$ is continuous except one uses the continuity of $r$ in Proposition II.5 instead of that of $\psi$. We will omit the details of this. Now consider the map $T: G_{\text {can }}(X) \times F H(X) \rightarrow G(X)$ defined by $T(h, g)=h \circ g$. We claim that $T$ is continuous. Suppose that $T(h, g) \in(K, V)$ in $G(X)$. Let $K^{\prime}=\{F(t, x):|t| \leqq f(x)$ and $x \in N$ with $F(q, x) \in K$ for some $(q, x) \in Z\} \cup K$. Now consider the space of all continuous functions from $K^{\prime}$ to $K^{\prime}, C\left(K^{\prime}, K^{\prime}\right)$. The set

$$
F H(X) \mid K^{\prime}=\left\{g \mid K^{\prime}: g \in F H(X)\right\}
$$

is a subset of $C\left(K^{\prime}, K^{\prime}\right)$. Consider the space of all continuous functions from $K^{\prime}$ to $X, C\left(K^{\prime}, X\right)$. Then composition is continuous from $C\left(K^{\prime}, X\right) \times C\left(K^{\prime}, K^{\prime}\right)$ $\rightarrow C\left(K^{\prime}, X\right)$ by [12, Theorem 2.2, p. 259]. Let $\left\{K_{i}\right\}_{i=1}^{n}$ and $\left\{L_{i}\right\}_{i=1}^{k}$ be collections of compact subsets of $K^{\prime}$ and $\left\{V_{i}\right\}_{i=1}^{n}$ and $\left\{U_{i}\right\}_{i=1}^{k}$ be collections of open sets in $X$ such 
that (1) $g \mid K^{\prime} \in \bigcap_{i=1}^{n}\left(K_{i}, V_{i} \cap K^{\prime}\right)$; (2) $h \mid K^{\prime} \in \bigcap_{i=1}^{k}\left(L_{i}, U_{i}\right)$; and (3) if $p$ $\in \bigcap_{i=1}^{n}\left(K_{i}, V_{i} \cap K^{\prime}\right)$ and $q \in \bigcap_{i=1}^{k}\left(L_{i}, U_{i}\right)$, then $q \circ p \in(K, V)$ for $p \in C\left(K^{\prime}, K^{\prime}\right)$ and $q \in C\left(K^{\prime}, X\right)$. Then we have that $g \in \bigcap_{i=1}^{n}\left(K_{i}, V_{i}\right), h \in \bigcap_{i=1}^{k}\left(L_{i}, U_{i}\right)$, and for $p \in F H(X)$ and $q \in G_{\text {can }}(X)$ with $p \in \bigcap_{i=1}^{n}\left(K_{i}, V_{i}\right)$ and $q \in \bigcap_{i=1}^{k}\left(L_{i}, U_{i}\right), q \circ p$ $\in(K, V)$. This implies that $T$ is continuous.

Now to show that $T$ is one-to-one. If $T(h, g)=q$, then using Proposition II.3 and Proposition II.5 we get that $J(q)=h$ and $K(q)=g$. If we define $P: G(X) \rightarrow G_{\text {can }}(X) \times F H(X)$ by $P(h)=(J(h), K(h))$, then $P$ is a homeomorphism and Claim 3 is now proved.

Claim 4. $F H(X) \sim F H(X) \times l_{2}$.

Proof of Claim 4. The set of fiber homeomorphisms of $X, F H(X)$, is homeomorphic as a function space to the space $F H(Z)$ defined by $h \in F H(Z)$ if and only if $h \in G(Z)$ and, for each $x \in N, h$ restricted to the $\operatorname{arc}[-f(x), f(x)] \times\{x\}$ is an orientation preserving homeomorphism of $[-f(x), f(x)] \times\{x\}$ onto itself. Now let $x_{0} \in Q$ be fixed. Recall the definition of $g_{x}:[0,1] \rightarrow[-f(x), f(x)] \times\{x\}$ for $x \in N$. Now for $h \in F H(Z)$ let $K(h)$ be defined by

$$
g_{x}^{-1} \circ K(h) \circ g_{x} \equiv\left(g_{x}^{-1} \circ h \circ g_{x}\right)\left(g_{x_{0}}^{-1} \circ h \circ g_{x_{0}}\right)^{-1}
$$

for all $x \in Q$. Let $K(h)(x)=x$ for $x \in N-Q$. Then one can easily verify that $K(h)$ $\in F H(Z)$ and that $K$ is continuous from $F H(Z)$ into $F H(Z)$. Now let $J(h)$ be such that $g_{x}^{-1} \circ J(h) \circ g_{x} \equiv g_{x_{0}}^{-1} \circ h \circ g_{x_{0}}$ for all $x \in Q$ and $J(h)(x)=x$ for $x \in N-Q$. Then $J$ is continuous with $J(h) \in F H(Z)$ also. Now the image of $J$ in $F H(Z)$ is homeomorphic to $H_{0}(I)$ by the map $L$ which takes $h \in H_{0}(I)$ to the map $L(h)$ defined by $g_{x}^{-1} \circ L(h) \circ g_{x} \equiv h$ for all $x \in Q$ and $L(h)(x)=x$ for $x \in N-Q$. Let $F H_{0}(Z)$ be the image of $H_{0}(I)$ under $L$ which is just the image of $F H(Z)$ under $K$. Let $F H_{1}(Z)$ be the image of $F H(Z)$ under $J$. Then the map $P: F H(Z) \rightarrow F H_{1}(Z) \times F H_{0}(Z)$ defined by $P(h)=(J(h), K(h))$ is a homeomorphism. The inverse of $P$ is just composition. But $F H_{0}(Z) \sim H_{0}(I)$ and thus $F H_{0}(Z) \sim l_{2}$ by Theorem III.1. Therefore $F H(Z) \times l_{2} \sim F H(Z)$. Thus $F H(X) \times l_{2} \sim F H(X)$ and Claim 4 is proved.

This last claim now completes the proof of Theorem III.2 which now follows from Claim 3 and Claim 4.

III.3. Corollary. Suppose that $X$ is separable and locally compact and admits a nontrivial flow. Then $\sigma$-compact subsets of $G(X)$ are negligible.

Proof. If $X$ is locally compact, then $G(X)$ is separable metric by [12, Theorem 5.2, p. 265]. By Theorem III.2, $G(X) \sim G(X) \times l_{2}$. By a theorem of Anderson in [1], this implies that $\sigma$-compact subsets of $G(X)$ are negligible.

III.4'. REMARK. This is a typical corollary to many of the theorems to follow. Once we have shown a certain function space $F(X, Y) \subset C(X, Y)$ to have the property that $F(X, Y) \sim F(X, Y) \times l_{2}$, then if $F(X, Y)$ is separable metric, then $\sigma$-compact subsets of $F(X, Y)$ are negligible. It is well known that separability and second countability are equivalent in metric spaces. Thus by [12, Theorem 5.2, 
p. 265] (together with [12, 13(b), p. 258] and Urysohn's metrization theorem [12, p. 195]), $F(X, Y)$ will be separable metric whenever $X$ is separable metric and locally compact and $Y$ is separable metric. We will not restate this corollary after many of the theorems, but the reader should be aware of this application.

III.4'. RemarK. As pointed out by the referee of this paper the proof of Corollary III.3 simply required that $G(X)$ be separable metric which is often the case even when $X$ is not separable and $\sigma$-compact. However, as he points out, a more general corollary is possible using Cutler's results in [11]. Cutler has shown that for all complete metric spaces $Y, \sigma$-compact subsets of $Y \times l_{2}$ are negligible (Lemma 1 and Theorem 1 of [11]). Thus if $G(X)$ is a complete metric space and $X$ admits a flow, then $\sigma$-compact subsets of $G(X) \times l_{2}$ are negligible. This remark has an application to many of the other theorems of this paper in the same way as the previous remark.

III.5. Remark. It is worth noting that even if $X$ is metric and admits a flow, $\sigma$-compact subsets of $G(X)$ may not be negligible. For example if one lets $X$ be the free topological union of uncountably many unit intervals $\left\{I_{\alpha}: \alpha \in A\right\}$. In this case $G(X)$ is a topological group and the identity component of $G(X)$ is homeomorphic to $\prod_{\alpha \in A} l_{2} \sim \prod_{\alpha \in A} R_{\alpha}$ by Theorem III.1. However, $\prod_{\alpha \in A} R_{\alpha}$ is realcompact and $\prod_{\alpha \in A} R_{\alpha}$ less any one point is not. Thus not even one point sets are negligible in this case.

We now want to make a few observations about the proof of Theorem III. 2 and point out that with only slight modification one can establish a number of other function space results. In the next section some additional function spaces will be dealt with and similar results obtained.

III.6. TheOREM. Suppose that $X$ admits a nontrivial flow and that $E(X, Y)$ is the space of all imbeddings of $X$ into the metric space $Y$. Then $E(X, Y) \sim E(X, Y) \times l_{2}$.

Proof. We could define $E_{\text {can }}(X, Y)$ in a manner analogous to our definition of $G_{\text {can }}(X)$. We could then define maps $J: E(X, Y) \rightarrow E_{\text {can }}(X, Y)$ and $K: E(X, Y)$ $\rightarrow F H(X)$ in a manner similar to the corresponding maps in the proof of Theorem III.2. With these modifications the proof would be essentially the same.

Note that if $e \in E(X, Y)$, then the image of $e$ is open (closed) in $Y$ if and only if the image of $J(e)$ is open (closed). Let us define $E_{\mathrm{cl}}(X, Y)$ to be the closed imbeddings of $X$ and $E_{\mathrm{op}}(X, Y)$ the open ones. The above remark implies that $T\left[J\left[E_{\mathrm{cl}}(X, Y)\right] \times F H(X)\right]=E_{\mathrm{cl}}$ and $T\left[J\left[E_{\mathrm{op}}(X, Y)\right] \times F H(X)\right]=E_{\mathrm{op}}(X, Y)$.

III.7. THEOREM. If $X$ admits a nontrivial flow and $Y$ is metric, then $E_{\mathrm{cl}}(X, Y)$ $\sim E_{\mathrm{cl}}(X, Y) \times l_{2}$ and $E_{\mathrm{op}}(X, Y) \sim E_{\mathrm{op}}(X, Y) \times l_{2}$.

To observe the next few theorems we need to observe our construction of the set $F[Z]$ in $X$ in the proof of Theorem III.2. If $x_{0}$ has a nondegenerate orbit under the flow $F$, then our construction of the set $F[Z]$ could have been required to be within any preassigned neighborhood of $x_{0}$. Using this fact one can easily verify the following results. 
III.8. Theorem. Let $A$ be a closed subset of $X$ and $B$ any subset of $X$. Suppose that $X$ admits a nontrivial flow. Let $G_{A, B}(X)=\{f \in G(X): f(A) \subset B\}$. Then $G_{A, B}(X)$ $\sim G_{A, B}(X) \times l_{2}$.

III.9. TheOREM. Suppose that $X$ admits a nontrivial flow and that $A$ is closed in $X$ and $B$ is any subset of $Y$, a metric space. Then if $E((X, A),(Y, B))$ is the function space of all imbeddings of the pair $(X, A)$ into the pair $(Y, B)$, then $E((X, A),(Y, B))$ $\sim E((X, A),(Y, B)) \times l_{2}$.

III.10. Theorem. Suppose that $X$ admits a nontrivial flow. Let $A$ be a closed subset of $X$ such that not all nondegenerate orbits of the flow are contained in $A$. Then let $G_{A}(X)=\{f \in G(X): f \mid A$ is the identity $\}$. Then $G_{A}(X) \sim G_{A}(X) \times l_{2}$.

III.11. Theorem. Suppose that $X$ admits a nontrivial flow. Let $A$ be closed in $X$ such that not all nondegenerate orbits of the flow are in $A$. Then let $f: X \rightarrow Y$ be a given imbedding of $X$ into $Y, Y$ a metric space. Then let

$$
E_{f, A}(X, Y)=\{g \in E(X, Y): g|A=f| A\} .
$$

Then $E_{f, A}(X, Y) \sim E_{f, A}(X, Y) \times l_{2}$.

We close this section with a similar theorem, but one which requires different techniques. As in [1] we let the Hilbert cube be the product $\prod_{i=1}^{\infty}[0,1]_{i}=I^{\infty}$ and the pseudoboundary of $I^{\infty}$ be the set of points $x \in I^{\infty}$ such that, for some coordinate $i, x_{i}=0$ or $x_{i}=1$. The pseudoboundary of $I^{\infty}$ is dense in $I^{\infty}$ and $\sigma$-compact.

III.12. THEOREM. If $X$ is the Hilbert cube and $G_{P}(X)$ is the space of homeomorphisms taking the pseudoboundary of $X$ onto the pseudoboundary of $X$, then $G_{P}(X) \sim G_{P}(X) \times l_{2}$.

Proof. Let $R_{\gamma}$ be the two point compactification of the real numbers $R$. Denote the two added points by $\infty$ and $-\infty$. Of course, $R_{\gamma} \sim[0,1]$ and $R_{\gamma}^{\infty} \sim I^{\infty}$. Consider $R_{y}^{\infty}$ as the space $X$ and the pseudoboundary of $X$ as the set of points $\left(x_{i}\right) \in R_{\gamma}^{\infty}$ such that, for some $j, x_{j}=\infty$ or $x_{j}=-\infty$. For each $x \in R$ there is a homeomorphism associated with $x$ on $R_{y}$ defined by $f_{x}(y)=x+y$ for $y \in R$ and $f_{x}(y)=y$ if $y=\infty$ or $y=-\infty$. The map $A: R \times R_{y} \rightarrow R_{\gamma}$ defined by $A(x, y)=f_{x}(y)$ is a flow on $R_{y}$. Let $A^{\infty}: R^{\infty} \times R_{\gamma}^{\infty} \rightarrow R_{\gamma}^{\infty}$ be defined by $A^{\infty}\left(\left(x_{i}\right),\left(y_{i}\right)\right)=\left(z_{i}\right)$ where $z_{i}=f_{x_{i}}\left(y_{i}\right)$. Then $A^{\infty}$ is a topological group action of $R^{\infty}$ on $R_{\gamma}^{\infty}$. Suppose now that $f \in G_{P}(X)$. Then let $J(f)=\left(x_{i}\right) \in R^{\infty}$ where $x_{i}$ is the $i$ th coordinate of $f((0,0, \ldots))$. Note that $J(f) \in R^{\infty}$ since $f \in G_{P}(X)$. Now with each $\left(x_{i}\right) \in R^{\infty}$, there is a homeomorphism of $R_{y}^{\infty}$ associated with it under $A^{\infty}$, namely, $Q\left(\left(x_{i}\right)\right)\left(\left(y_{i}\right)\right)=A^{\infty}\left(\left(x_{i}\right),\left(y_{i}\right)\right)$. Of course $Q\left(\left(x_{i}\right)\right) \in G_{P}(X)$ for all $\left(x_{i}\right) \in R^{\infty}$. Now let $K(f) \in G_{P}(X)$ be defined by $K(f)$ $=Q(-J(f)) \circ f$. Then if $G_{P, 0}(X)=\left\{f \in G_{P}(X): f((0,0, \ldots))=(0,0, \ldots)\right\}$, then $K(f) \in G_{P, 0}(X)$. Define $P: G_{P}(X) \rightarrow R^{\infty} \times G_{P, 0}(X)$ by $P(f)=(J(f), K(f))$. Then it is routine, if tedious, to verify that $P$ is a homeomorphism. The inverse of $P$ is just $P^{-1}\left(\left(x_{i}\right), h\right)=Q\left(\left(x_{i}\right)\right) \circ h$. Now by [3], $R^{\infty} \sim l_{2}$. Therefore $G_{P}(X) \sim G_{P}(X) \times l_{2}$ and the theorem is proved. 
III.13. Corollary. Let $X$ be the Hilbert cube. Then $\sigma$-compact subsets of $G_{P}(X)$ are negligible.

4. Other function spaces. In this section we use the same techniques as in the proof of Theorem III.2 to obtain results about other function spaces having Hilbert space as a factor. We will assume throughout this section that $X$ and $Y$ are metric spaces. Let $g: X \rightarrow Y$ be continuous. We will say that $g$ is totally arcwise disconnected if for all $y \in Y, g^{-1}(y)$ contains no nontrivial arcs. An example of such a map would be a light map, that is, a $g$ such that $g^{-1}(y)$ is totally disconnected for all $y \in Y$. Actually, $g$ could have continuum point inverses and still be totally arcwise disconnected, for instance if all the point inverses of $g$ were pseudoarcs. What is important in this section is the application of our results to light open mappings. Let $\operatorname{TAD}(X, Y)$ denote the totally arcwise disconnected maps from $X$ to $Y$.

IV.1. Theorem. Suppose that $X$ admits a nontrivial flow. Then $\operatorname{TAD}(X, Y)$ $\sim \operatorname{TAD}(X, Y) \times l_{2}$.

Proof. Let $F$ be a flow on $X$ and let $x_{0}$ be a point having a nondegenerate orbit under $F$. Recall the construction of the flow $F^{\prime}$ in the proof of Theorem III.2. As in that proof call the new flow $F$ since we will not need our original flow again. Recall our construction of the local cross-section $N$ through $x_{0}$ and of the set $Z \subset[-1,1] \times N$. Recall that $Q=$ int $_{X} F[[-1,1] \times N] \cap N$. Now for each $x \in Q$ let $g_{x}:[0,1] \rightarrow[-f(x), f(x)] \times\{x\}$ be linear with $g_{x}(0)=(-f(x), x)$ and $g_{x}(1)$ $=(f(x), x)$. Now if $g \in \operatorname{TAD}(X, Y)$, then the map $g \circ F \circ g_{x}:[0,1] \rightarrow Y$ has the property that it is constant on no interval for all $x \in Q$. That is, $g \circ F \circ g_{x} \in D(I, Y)$. Now define the maps $J: \operatorname{TAD}(X, Y) \rightarrow \operatorname{TAD}(X, Y)$ and $K: \operatorname{TAD}(X, Y) \rightarrow F H(X)$ by the conditions that $K(g) \mid X-$ int $_{X} F[Z]$ is the identity and for $x \in Q, K(g)$ on the fiber through $x$ is defined by the condition that $g_{x}^{-1} \circ F^{-1} \circ K(g) \circ F \circ g_{x}$ $\equiv r\left(g \circ F \circ g_{x}\right)$ where $r$ is as in $\S 2$ of the paper. Now $K(g)$ can be shown to be a fiber homeomorphism and $K: \operatorname{TAD}(X, Y) \rightarrow F H(X)$ can be shown to be continuous. One uses the results in $\S 2$ and the techniques used in the proof of Theorem III. 2 to show this. Now define $J(g)$ as $J(g) \mid X-$ int $_{X} F[Z] \equiv g$ and for $x \in Q, J(g)$ is defined on the fiber through $x$ by $J(g) \circ F \circ g_{x} \equiv \psi\left(g \circ F \circ g_{x}\right)$. Then let $\operatorname{TAD}_{\text {can }}(X, Y)$ be the image of $\operatorname{TAD}(X, Y)$ under $J$. Then $J$ is continuous and the map $P: \operatorname{TAD}(X, Y) \rightarrow \mathrm{TAD}_{\text {can }}(X, Y) \times F H(X)$ defined by $P(g)=(J(g), K(g))$ is a homeomorphism. The details are similar to the proof of Theorem III.1. Since $F H(X) \sim F H(X) \times l_{2}$ we then have that TAD $(X, Y) \sim$ TAD $(X, Y) \times l_{2}$ as asserted.

What is useful is that the map $P$ in the proof of Theorem IV.1 induces factorizations of other subspaces of $\operatorname{TAD}(X, Y)$ as well. Actually, if $X=Y$, then $G(X)$ $\subset$ TAD $(X, Y)$ and the $P$ in the proof of Theorem IV.1 restricted to $G(X)$ is the $P$ in the proof of Theorem III.2. Let us denote the light mappings from $X$ to $Y$ by $\mathrm{L}(X, Y)$. Let the light open and the light closed maps be denoted by $\mathrm{LO}(X, Y)$ and $\mathrm{LCl}(X, Y)$, respectively. 
IV.2. THEOREM. Suppose that $X$ admits a nontrivial flow. Then the following holds: $\mathrm{L}(X, Y) \sim \mathrm{L}(X, Y) \times l_{2}, \mathrm{LO}(X, Y) \sim \mathrm{LO}(X, Y) \times l_{2}$, and $\mathrm{LCl}(X, Y) \sim \mathrm{LCl}(X, Y)$ $\times l_{2}$.

IV.3. Corollary. Suppose that $X$ admits a nontrivial flow and that $X$ is separable and locally compact and that $Y$ is separable. Then $\sigma$-compact subsets of each of the following function spaces are negligible: $\operatorname{TAD}(X, Y), \mathrm{L}(X, Y), \mathrm{LO}(X, Y)$, and $\mathrm{LCl}(X, Y)$.

IV.4. Corollary. Let $X$ be a manifold and $Y$ be arbitrary. Then $\mathrm{L}(X, Y)$ $\sim \mathrm{L}(X, Y) \times l_{2}, \mathrm{LO}(X, Y) \sim \operatorname{LO}(X, Y) \times l_{2}$, and $\mathrm{LCl}(X, Y) \sim \mathrm{LCl}(X, Y) \times l_{2}$ and each of these function spaces has the property that $\sigma$-compact subsets are negligible whenever $X$ and $Y$ are both separable.

IV.5. REMARK. There are any number of other function spaces $F(X, Y)$ for which our techniques apply to give $F(X, Y) \sim F(X, Y) \times l_{2}$. For instance $F(X, Y)$ could be the finite to one open mappings, the finite to one closed mappings, the countable to one mappings, the monotone mappings with hereditarily indecomposable point inverses, etc. Our list could be long.

5. Spaces which admit a flow. Here we will investigate a few of the spaces $X$ for which the theorems of $\S 3$ and $\S 4$ apply. Basically we show various ways that flows may be induced on a space. Let $\mathscr{B}$ be the class of metric spaces which admit a nontrivial flow. Suppose that $X \in \mathscr{B}$ and that $Y$ is a metric space. Then $X \times Y \in \mathscr{B}$ also. Thus $G(X \times Y) \sim G(X \times Y) \times l_{2}$ by Theorem III.2.

V.1. TheOREM. If $X$ contains an open set $V$ with $V \in \mathscr{B}$, then $X \in \mathscr{B}$.

Proof. Let $x \in V$ have a nontrivial orbit under the flow $F$ on $V$. Let $x \in U$ with $U$ open such that $\bar{U} \subset V$ and such that for all $y \in U$, the orbit of $y$ under $F$ is nondegenerate. Then there is a flow $F^{\prime}$ on $V$ having as its set of fixed points the set $V-U$ and such that for each $y \in U$, the orbit of $y$ under $F^{\prime}$ is nondegenerate. The flow $F^{\prime}$ can be extended to all of $X$ by defining $F^{\prime}(t, x)=x$ for all $x \notin V$. Then $F^{\prime}$ will be a nontrivial flow on $X$.

V.2. COROLlaRY. If $X$ contains a subset $Y$ with nonempty interior such that $Y$ is homeomorphic to $R^{n}$ or $l_{2}$ or $I^{\infty}$, then $X \in \mathscr{B}$.

V.3. COROLlaRY. If $X$ contains an open set $V$ such that $\bar{V}$ is homeomorphic to the cone of $\bar{V}-V$, then $X \in \mathscr{B}$.

Proof. The cone of any space admits a nontrivial flow.

This implies that all spaces admitting a cone patch are in $\mathscr{B}$ and that Theorem III. 2 is a proper generalization of the results in [15, §2].

V.4. THEOREM. If $X$ is a topological group which admits a nontrivial one parameter subgroup, then $X \in \mathscr{B}$. 
Proof. Let $f: R \rightarrow X$ be a nontrivial one parameter subgroup. Define $F: R \times X \rightarrow X$ by $F(t, x)=f(t) \cdot x$. Then $F$ is a nontrivial flow.

V.5. COROLlaRY. If $X$ is a locally compact topological group which is not totally disconnected, then $X \in \mathscr{B}$.

Proof. This is well known. See for instance [26, p. 175, and Theorem 1, p. 192].

V.6. ExAmpLE. An application of Corollary V.5 is that if $X$ is a solenoid, then $G(X) \sim G(X) \times l_{2}$. Another example is the infinite torus. Now if we have any locally compact topological group $X$, we have that $G(X) \sim X \times P$ where

$$
P=\{f \in G(X): f(e)=e\}
$$

where $e$ is the identity of the group $X$ and $X=\left\{L_{x}: x \in X\right\}$ where $L_{x} \in G(X)$ is the homeomorphism $L_{x}(y)=x \cdot y$ for all $y \in X$. The homeomorphism between $G(X)$ and $X \times P$ is just $H: G(X) \rightarrow X \times P$ defined by $H(f)=\left(L_{f(e),}, L_{f(e)}^{-1} \circ f\right)$. The inverse of $H$ is just composition. This implies that for $X$ a solenoid and not the circle or for $X$ the infinite torus, $G(X)$ is not a separable Fréchet manifold. Actually in each of these cases $G(X)$ has $2^{\aleph_{0}}$ arc components.

V.7. TheOREM. If $X \in \mathscr{B}$ and $\mathscr{C}(X)=\{K \in X: K$ is compact $\}$ where $\mathscr{C}(X)$ has the finite topology, then $\mathscr{C}(X) \in \mathscr{B}$.

Proof. The finite topology is defined in [24, Definition 1.7]. We have that $\mathscr{C}(X)$ is metrizable by [24]. Let $F$ be a nontrivial flow on $X$. Then define

$$
\hat{F}: R \times \mathscr{C}(X) \rightarrow \mathscr{C}(X)
$$

by $\hat{F}((t, K))=F[\{t\} \times K]$. One can easily verify that $\hat{F}$ is a nontrivial flow on $\mathscr{C}(X)$.

V.8. Remark. Notice that $\hat{F}$ induces a nontrivial flow on various subspaces of $\mathscr{C}(X)$ as well, for instance on $\mathscr{F}_{n}(X)$, the subsets of $X$ having at most $n$ points, $\mathscr{F}(X)$, the finite subsets of $X$, and the continuum subsets. Let $\mathscr{P}$ be a topological property and let $\mathscr{P}(X)=\{K \in \mathscr{C}(X): K$ has $\mathscr{P}\}$. Then $\hat{F}$ is a flow on $\mathscr{P}(X)$ as well, but perhaps trivial. The set $\mathscr{P}(X)$ could be the countable and compact sets, the arcs, the pseudoarcs, etc. The list could be long.

V.9. Example. If $I$ is the unit interval then $G\left(2^{I}\right) \sim G\left(2^{I}\right) \times l_{2}$. This is another property which $2^{I}$ has in common with $I^{\infty}$. A long outstanding problem has been whether $2^{I}$ is homeomorphic to $I^{\infty}$.

V.10. Example. Suppose that $X$ admits a nontrivial flow $F$. Let

$$
\hat{F}: R \times \mathscr{C}(X) \rightarrow \mathscr{C}(X)
$$

Let $L \in \mathscr{C}(X)$ be a compact subset of $X$ which is not a singleton and which has a nondegenerate orbit under $\hat{F}$. Let $K \neq L$ in $\mathscr{C}(X)$ be on the orbit of $L$ under $\hat{F}$ in $\mathscr{C}(X)$. Clearly, if $A(L, K)$ is an arc joining $L$ and $K$ lying in the orbit of $L$ under $F$, then $A(L, K)$ does not intersect the set of singletons $\{\{x\}: x \in X\}$ which is closed in $\mathscr{C}(X)$. Let $\mathscr{V}$ be open in $\mathscr{C}(X)$ such that $\mathscr{V}$ contains $A(L, K)$ and for every $C \in \mathscr{V}$, 
the orbit of $C$ under $\hat{F}$ is nondegenerate. Then there is a flow $\hat{F}^{\prime}$ on $\mathscr{C}(X)$ such that the set of fixed points of $\hat{F}^{\prime}$ is just $\mathscr{C}(X)-\mathscr{V}$. In the construction of $F^{\prime}$, the orbit of $L$ under $F^{\prime}$ will still contain $K$. Thus there will be a $t \in R$ such that $F^{\prime}(t, L)=K$. Let $g$ be the homeomorphism on $\mathscr{C}(X)$ defined by $g(C)=\hat{F}^{\prime}(t, C)$. Then $g(L)=K$ and for every $x \in X, g(\{x\})=\{x\}$. That is, using flows we have a way of constructing homeomorphisms on $\mathscr{C}(X)$ which are not the identity, but which are the identity on the set of singletons. This technique also works for various subspaces of $\mathscr{C}(X)$ as well.

6. Some $G(X)$ which are separable Fréchet manifolds. A separable Fréchet manifold is a separable metric space which is locally homeomorphic to $l_{2}$. What we show in this section is that if $X$ is the familiar $\sin (1 / x)$-curve, then $G(X)$ is a separable Fréchet manifold. We also show that for $X$ the circle $1 / x$-curve, to be defined later, $G(X)$ is homeomorphic to $l_{2}$.

There are a few lemmas which we will need in the subsequent proofs which we first state and prove.

VI.1. Theorem. Any two separable infinite-dimensional Banach spaces are homeomorphic.

This theorem is due to Kadeč (see [19] and [20]).

VI.2. Lemma. Let $X$ be compact metric and $B$ be a separable infinite-dimensional Banach space. Then $C(X, B)$ is a separable infinite-dimensional Banach space and thus homeomorphic to $B$. If $x \in X$ and $C_{x}(X, B)=\{f \in C(X, B): f(x)=0\}$, then $C_{x}(X, B)$ is also a separable infinite-dimensional Banach space and homeomorphic to $B$.

Proof. Let \|\|$_{B}$ be the norm of $B$. Define the norm of $C(X, B)$ to be $\|f\|$ $=\sup \left\{\|f(z)\|_{B}: z \in X\right\}$. Let addition and scalar multiplication be defined pointwise in $C(X, B)$. Then $C(X, B)$ becomes a separable infinite-dimensional Banach space with this norm and these operations. Also $C_{x}(X, B)$ is a closed infinite-dimensional subspace. By Theorem VI.1 both of these spaces are homeomorphic to $B$.

Now let $x_{i} \rightarrow 0$ be a sequence of real numbers with $x_{i}>x_{i+1}$ for all $i$. Then let $X$ be the set of $(x, y)$ in $R^{2}$ such that $x=0$ and $-1 \leqq y \leqq 1$ or $(x, y)$ lies on one of the segments joining $\left(x_{2 i-1},-1\right)$ to $\left(x_{2 i}, 1\right)$ or one of the segments joining $\left(x_{2 i}, 1\right)$ to $\left(x_{2 i-1},-1\right)$ for some $i$. Then $X$ is homeomorphic to the $\sin (1 / x)$-curve. Now let $H(X)=\left\{h \in G(X): h\left(\left(x_{2 i-1},-1\right)\right)=\left(x_{2 i-1},-1\right)\right.$ and $h\left(\left(x_{2 i}, 1\right)\right)=\left(x_{2 i}, 1\right)$ for all $\left.i\right\}$ and let $H_{0}(X)=\{h \in H(X): h \mid\{0\} \times[-1,1]$ is the identity $\}$.

VI.3. Proposition. The spaces $H(X)$ and $H_{0}(X)$ are homeomorphic to $l_{2}$.

Proof. Let $P$ be the positive integers and let $P^{*}=P \cup\{\infty\}$ be the one point compactification of $P$. Now let $h \in H(X)$ and $i \in P$. Let $f_{i}$ be defined on the segment joining $\left(x_{i},-1\right)$ to $\left(x_{i+1}, 1\right)$ for $i$ odd and on the segment joining $\left(x_{i}, 1\right)$ to $\left(x_{i+1},-1\right)$ 
for $i$ even and defined by $f_{i}((x, y))=y$. Then let $h_{i}=f_{i} \circ h \circ f_{i}^{-1}$. Then $h_{i} \in H_{0}(I)$ $=\{$ the orientation preserving homeomorphisms of $I=[-1,1]\}$. Let $f_{\infty}$ be defined on $\{0\} \times[-1,1]$ by $f_{\infty}((0, y))=y$. Then let $h_{\infty}=f_{\infty} \circ h \circ f_{\infty}^{-1}$. Then again $h_{\infty} \in H_{0}(I)$. Now let $F: H(X) \rightarrow C\left(P^{*}, H_{0}(I)\right)$ be defined by $F(h)(i)=h_{i}$ and $F(h)(\infty)=h_{\infty}$. Then $F$ is a homeomorphism of $H(X)$ onto $C\left(P^{*}, H_{0}(I)\right)$ and $F$ takes $H_{0}(X)$ onto $C_{\infty}\left(P^{*}, H_{0}(I)\right)$. By Theorem III.1 we have that $H_{0}(I) \sim l_{2}$. Therefore $H(X)$ and $H_{0}(X)$ are homeomorphic to $l_{2}$ by Lemma VI.2.

VI.4. Definition. Let $X$ be the set of points in $R \times R^{2}=R^{3}$ which are the closure of the points $\{(x, \exp (i / x)): 0<x \leqq 1 / 2 \pi\}$. Let $x_{n} \in X$ be the point $(1 / 2 n \pi, 1,0)=(1 / 2 n \pi$, exp $(2 n \pi i))$. Let $H(X)$ be the homeomorphisms $h$ of $X$ such that $h\left(x_{n}\right)=x_{n}$ for all $n$. Let $H_{0}(X)$ be the subgroup of $H(X)$ such that $h \mid\{0\} \times S^{1}$ is the identity. We will call the space $X$ the circle $1 / x$-curve.

VI.5. Proposition. Let $X$ be the circle $1 / x$-curve. Then $H(X)$ and $H_{0}(X)$ are homeomorphic to $l_{2}$.

Proof. We first note that if $H_{0}\left(S^{1}\right)$ is the group of orientation preserving homeomorphisms $h$ of the unit circle such that $h(1)=1$, then $H_{0}\left(S^{1}\right) \sim l_{2}$ by Theorem III.1. Now let $i \in P$ and let $S_{i}^{1}$ be the points in $X$ whose first coordinate lies between $1 / 2(i+1) \pi$ and $1 / 2 i \pi$. Let $f_{i}: S_{i}^{1} \rightarrow S^{1}$ be defined by $f_{i}((x, y))=y$ for $(x, y) \in S_{i}^{1}$. Then for $h \in H(X)$ let $h_{i}$ be defined by $h_{i}=f_{i} \circ h \circ f_{i}^{-1}$. Then $h_{i}$ is well defined and $h_{i} \in H_{0}\left(S^{1}\right)$. Let $f_{\infty}:\{0\} \times S^{1} \rightarrow S^{1}$ be defined by $f_{\infty}((0, y))=y$. Then let $h_{\infty}$ $=f_{\infty} \circ h \circ f_{i}^{-1}$. Then $h_{\infty} \in H_{0}\left(S^{1}\right)$ also. Define $F: H(X) \rightarrow C\left(P^{*}, H_{0}\left(S^{1}\right)\right)$ by $F(h)(i)=h_{i}$ and $F(h)(\infty)=h_{\infty}$. Then $F$ is a homeomorphism onto and $F$ takes $H_{0}(X)$ onto $C_{\infty}\left(P^{*}, H_{0}\left(S^{1}\right)\right)$. Therefore $H(X)$ and $H_{0}(X)$ are homeomorphic to $l_{2}$ by Lemma VI.2.

The next lemma is a routine result in function space topology which we will not prove. We simply want to isolate it for reference.

VI.6. Lemma. Suppose that $X$ and $Y$ are compact metric spaces and that $\left\{f_{i}\right\}$ is a sequence of continuous functions from $X$ into $Y$. Let $f \in C(X, Y)$. Then $\left\{f_{i}\right\}$ does not converge uniformly to $f$ if and only if there is a sequence $x_{j} \rightarrow x$ in $X$ and a subsequence $\left\{f_{i,}\right\}$ of $\left\{f_{i}\right\}$ such that $f_{i,}\left(x_{j}\right) \rightarrow y \neq f(x)$.

If the sine function is restricted to the interval $[\pi / 2+k \pi, \pi / 2+(k+1) \pi]$ where $k$ is an integer, then it is one-to-one and continuous onto $[-1,1]$. Therefore it has an inverse, the arcsine function. We will identify this branch of the arcsine function as the $k$-branch. We will be mainly interested in the 0 -branch mapping $[-1,1]$ onto $[\pi / 2,3 \pi / 2]$ and the 1-branch mapping $[-1,1]$ onto $[3 \pi / 2,5 \pi / 2]$. Now suppose that $x, y \in[\pi / 2+k \pi, \pi / 2+(k+1) \pi]$ and $z, w \in[\pi / 2+m \pi, \pi / 2+(m+1) \pi]$. Then if $\sin x=\sin z$ and $\sin y=\sin w$, then for any $0 \leqq t \leqq 1, \quad \sin (t x+(1-t) y)$ $=\sin (t z+(1-t) w)$. This implies that for any $k$-branch of the arcsine, if $a, b$ $\in[-1,1]$, then $\sin (t(\arcsin a)+(1-t)(\arcsin b))$ is independent of $k$ for all $t \in[0,1]$. 
VI.7. Lemma. For all $a, b \in[-1,1]$ and all $t \in[0,1]$,

$$
\sin (t(\arcsin a)+(1-t)(\arcsin b))
$$

is independent of the choice of $k$-branch for the arcsine function.

We are now prepared to state the first main theorem in this section.

VI.8. TheOREM. Let $X$ be the $\sin (1 / x)$-curve. Then $G(X)$ is homeomorphic to $l_{2} \times N$ where $N$ is the integers.

Proof. Let $R^{+}=\{x \in R: x \geqq 0\}$. We will describe $X$ as a compactification of $R^{+}$. Basically $X$ will be the minimum compactification of $R^{+}$allowing an extension of the sine function. We will think of $X$ as $R^{+}$together with the added points $A=\{\bar{\alpha}: \alpha \in[-1,1]\}$. For $\alpha \in[-1,1]$, a neighborhood basis for $\bar{\alpha}$ is the collection of all sets of the form

$$
O(r, \varepsilon, \alpha)=\left\{x \in R^{+}: x>r \text { and }|\sin x-\alpha|<\varepsilon\right\} \cup\{\bar{\beta} \in A:|\beta-\alpha|<\varepsilon\}
$$

where $r \in R^{+}$and $\varepsilon>0$. Let us observe at this point that if $f \in G(X)$, then $f\left[R^{+}\right]$ $=R^{+}$and $f[A]=A$. Now define $h: G(X) \rightarrow N$ by

$$
h(f)=\frac{1}{\pi} \lim _{k \rightarrow \infty}\left[f\left(2 k \pi+\frac{\pi}{2}\right)-\left(2 k \pi+\frac{\pi}{2}\right)\right] .
$$

Claim 1. The function $h$ is well defined and a topological group homomorphism onto the integers.

Proof of Claim 1. Since $f$ is a homeomorphism of $X, f(\overline{1})=\overline{1}$ or $f(\overline{1})=-\overline{1}$. Let $\bar{\gamma}=f(\overline{1})$. Now since $2 k \pi+\pi / 2 \rightarrow \overline{1}$ in $X$, we must have that $f(2 k \pi+\pi / 2) \rightarrow \bar{\gamma}$. Therefore there is an integer $K$ such that for $k \geqq K, f(2 k \pi+\pi / 2) \in O\left(1, \frac{1}{2}, \gamma\right)$. Then for $k \geqq K$ we have that $f(2 k \pi+\pi / 2) \in\left(\pi n_{k}+\pi / 2-\pi / 3, \pi n_{k}+\pi / 2+\pi / 3\right)$ for some integer $n_{k}$. We claim that for some $L \geqq K$ it is true that for all $k \geqq L, n_{k+1}=n_{k}+2$. If this were not so, one could construct a sequence $x_{i} \in R^{+}$such that $x_{i} \rightarrow \infty$, $\left|\sin x_{i}-1\right| \geqq \varepsilon>0$ with $\sin f\left(x_{i}\right)=\gamma$ for all $i$. This would contradict the continuity of $f^{-1}$ on $X$. Now since $f(2 k \pi+\pi / 2) \rightarrow \bar{\gamma}$ we get that for $k \geqq L, f(2 k \pi+\pi / 2)$ $=n_{k} \pi+\pi / 2+\varepsilon_{k}$ where $\varepsilon_{k} \rightarrow 0$ as $k \rightarrow \infty$. Thus for $k \geqq L$,

$$
(1 / \pi)[f(2 k \pi+\pi / 2)-(2 k \pi+\pi / 2)]=n_{k}+2 k+\varepsilon_{k} / \pi=n_{L}-2 L+\varepsilon_{k} / \pi .
$$

Therefore $h(f)=n_{L}-2 L$ and this is an integer.

Suppose now that $f_{i} \rightarrow f_{0}$ in $G(X)$ and that $h\left(f_{i}\right)=n_{i}$ for all $i$. Let $f_{0}(\overline{1})=\bar{\gamma}$ where $\gamma=1$ or $\gamma=-1$. Let $K$ be an integer such that for all integers $k \geqq K, f_{0}(2 k \pi+\pi / 2)$ $\in\left(\pi n_{k}+\pi / 2-\pi / 3, \pi n_{k}+\pi / 2+\pi / 3\right)$ with $n_{k+1}=n_{k}+2$. We claim that there is an integer $P$ such that for all $i \geqq P, f_{i}(2 k \pi+\pi / 2) \in\left(\pi n_{k}+\pi / 2-\pi / 3, \pi n_{k}+\pi / 2+\pi / 3\right)$ for all $k \geqq L$ for some integer $L \geqq K$. If not, then there is a subsequence $\left\{f_{i}\right\}$ and a sequence $\left\{k_{j}\right\}$ such that $f_{i,}\left(2 k_{j} \pi+\pi / 2\right) \rightarrow-\bar{\gamma}$ which would contradict Lemma VI.4. But then for all $i \geqq P$ it is clear that $h\left(f_{i}\right)=n_{L}-2 L=h\left(f_{0}\right)$. Thus $h$ is continuous. 
To show that $h$ is a homomorphism of the group $G(X)$ into the integers $N$ we note that we could have defined $h$ in the following equivalent manner:

$$
h(f)=\frac{1}{\pi} \lim _{k \rightarrow \infty}\left[f\left(\left(k+\varepsilon_{k}\right) \pi+\frac{\pi}{2}\right)-\left(k \pi+\frac{\pi}{2}\right)\right]
$$

where $\varepsilon_{k} \rightarrow 0$ as $k \rightarrow \infty$. For any sequence $\varepsilon_{k} \rightarrow 0$, the limit will be the same. Now suppose that $h(f)=n_{f}$ and $h(g)=n_{g}$. Then $f(k \pi+\pi / 2)=\left(k+n_{f}+\varepsilon_{k}\right) \pi+\pi / 2$ where $\varepsilon_{k} \rightarrow 0$. Thus $g \circ f(k \pi+\pi / 2)=g\left(\left(k+n_{f}+\varepsilon_{k}\right) \pi+\pi / 2\right)=\left(k+n_{f}+n_{g}\right) \pi+\delta_{k}$ where $\delta_{k} \rightarrow 0$ as $k \rightarrow \infty$. Thus

$$
\frac{1}{\pi} \lim _{k \rightarrow \infty}\left[g \circ f\left(k \pi+\frac{\pi}{2}\right)-\left(k \pi+\frac{\pi}{2}\right)\right]=n_{g}+n_{f} .
$$

That is, $h(g \circ f)=h(g)+h(f)$.

To show that $h$ is onto let $n>0$ be an integer. We define $f_{n}: X \rightarrow X$ by the following formula for $x \in R^{+}$:

$$
\begin{aligned}
f_{n}(x) & =x(n+1), & & 0 \leqq x \leqq \pi, \\
& =x+n \pi, & & x \geqq \pi .
\end{aligned}
$$

Then for $\beta \in[-1,1]$ define $f_{n}(\bar{\beta})=\bar{\beta}$ for $n$ even and for $n$ odd let $f_{n}(\bar{\beta})$ $=\sin ((3 \pi / 2-\arcsin \beta)+\pi / 2)$ where we use the 0 -branch of the arcsine function. Then using Lemma VI.7 one can show that $f_{n}$ is continuous from $X$ onto $X$ for $n$ odd. It is easier to see that $f_{n}$ is continuous for $n$ even. Clearly $f_{n}$ is one-to-one and thus a homeomorphism of $X$ onto $X$. Now $h\left(f_{n}\right)=n$ and $h\left(f^{-1}\right)=-n$ and thus $h$ is onto.

Claim 2. The subgroup ker $h$ of $G(X)$ is contractible.

Proof of Claim 2. By the definition of $h$,

$$
\operatorname{ker} h=\left\{f \in G(X): \lim _{k \rightarrow \infty}\left[f\left(2 k \pi+\frac{\pi}{2}\right)-\left(2 k \pi+\frac{\pi}{2}\right)\right]=0\right\} .
$$

Let $t \in[0,1]$ and $f \in \operatorname{ker} h$. Then define $H(t, f)(x)=t x+(1-t) f(x)$ for $x \in R^{+}$and for $\alpha \in[-1,1]$ with $\bar{\beta}=f(\bar{\alpha})$ let $H(t, f)(\bar{\alpha})=\sin (t \arcsin \alpha+(1-t) \arcsin \beta)$ for some choice of $k$-branch for the arcsine function. Then by Lemma VI.7, $H(t, f)$ is well defined. Now $H(t, f)$ is clearly one-to-one and onto. Using Lemma VI.7 one can verify that $H(t, f): X \rightarrow X$ is also continuous. Therefore $H(t, f) \in G(X)$. We will now show that $H:[0,1] \times \operatorname{ker} h \rightarrow \operatorname{ker} h$ is continuous. We will then have proved that ker $h$ is contractible. Suppose that $H$ is not continuous. Then there is a sequence $t_{i} \rightarrow t_{0}$ and $f_{i} \rightarrow f_{0}$ such that $H\left(t_{i}, f_{i}\right) \rightarrow H\left(t_{0}, f_{0}\right)$. By Lemma VI.6, there is a sequence $x_{j} \rightarrow x_{0}$ in $H\left(t_{i j}, f_{i}\right)\left(x_{j}\right) \rightarrow y \neq H\left(t_{0}, f_{0}\right)\left(x_{0}\right)$. Let us resubscript so that $H\left(t_{i}, f_{i}\right)\left(x_{i}\right) \rightarrow y$. It should be clear that we can take $x_{i} \in R^{+}$for all $i$ and $x_{0}=\bar{\alpha} \in A$.

Case (i). $\bar{\alpha}=\overline{1}$ or $\bar{\alpha}=-\overline{1}$.

We will treat the case $\bar{\alpha}=\overline{1}$. The case $\bar{\alpha}=-\overline{1}$ is similar. Since $f_{i} \rightarrow f_{0}$ uniformly we must have $f_{i}\left(x_{i}\right) \rightarrow \overline{1}$ by Lemma VI.6. Since each $f_{i} \in \operatorname{ker} h$, this implies that 
$\left|x_{i}-f_{i}\left(x_{i}\right)\right| \rightarrow 0$. But then $\left|x_{i}-\left(t_{i} x_{i}+\left(1-t_{i}\right) f_{i}\left(x_{i}\right)\right)\right| \rightarrow 0$ also. That is,

$$
\left|x_{i}-H\left(t_{i}, f_{i}\right)\left(x_{i}\right)\right| \rightarrow 0 \text { and } H\left(t_{i}, f_{i}\right)\left(x_{i}\right) \rightarrow \overline{1} \text {, }
$$

a contradiction. Therefore $\bar{\alpha} \neq \overline{1}$. Also $\bar{\alpha} \neq-\overline{1}$.

Case (ii). $\alpha \in(-1,1)$.

Then let $x_{i}=z_{i}+2 k_{i} \pi$ where $\pi / 2 \leqq z_{i} \leqq 5 \pi / 2$. Also let $f\left(x_{i}\right)=y_{i}+2 p_{i} \pi$ where $\pi / 2 \leqq y_{i} \leqq 5 \pi / 2$ and let $f_{i}\left(x_{i}\right)=w_{i}+2 q_{i} \pi$ where $\pi / 2 \leqq w_{i} \leqq 5 \pi / 2$. There is an integer $P$ such that for $i \geqq P$ we have that $x_{i}, y_{i}$, and $w_{i}$ are in the open interval $(\pi / 2,5 \pi / 2)$. By the continuity of $h$ we have that $\left|w_{i}-y_{i}\right| \rightarrow 0$ and from some point on $p_{i}=k_{i}$ $=q_{i}$. We will assume that this is true for $i \geqq P$. We may also assume that for $i \geqq P$ that $z_{i}, y_{i}, w_{i} \neq 3 \pi / 2$. If $f(\bar{\alpha})=\bar{\beta}$, then $\sin z_{i} \rightarrow \alpha, \sin y_{i} \rightarrow \beta$ and also $\sin w_{i} \rightarrow \beta$. We now use Lemma VI.7. Now $H\left(t_{i}, f_{i}\right)\left(x_{i}\right)=t_{i} x_{i}+\left(1-t_{i}\right) f_{i}\left(x_{i}\right)$ and

$$
\begin{aligned}
\lim _{i \rightarrow \infty} H\left(t_{i}, f_{i}\right)\left(x_{i}\right) & =\lim _{i \rightarrow \infty} \sin \left(t_{i} x_{i}+\left(1-t_{i}\right) f_{i}\left(x_{i}\right)\right) \\
& =\lim _{i \rightarrow \infty} \sin \left(t_{i} z_{i}+\left(1-t_{i}\right) w_{i}\right)=\lim _{i \rightarrow \infty} \sin \left(t_{i} z_{i}+\left(1-t_{i}\right) y_{i}\right) \\
& =\sin \left(t_{0} \arcsin \alpha+\left(1-t_{0}\right) \arcsin \beta\right)=H\left(t_{0}, f_{0}\right)(\bar{\alpha}),
\end{aligned}
$$

again a contradiction. Therefore $H$ is continuous and $\operatorname{ker} h$ is contractible.

Claim 3. The space ker $h$ is a separable Fréchet manifold.

Proof of Claim 3. Since ker $h$ is a topological group we only need to show that there is one open set in $\operatorname{ker} h$ homeomorphic to $l_{2}$. Let

$$
O=\left\{f \in \operatorname{ker} h: f\left(k \pi+\frac{\pi}{2}\right) \in(k \pi,(k+1) \pi)\right\} .
$$

Now $O$ is open in $G(X)$ and we will proceed to show that $O \sim l_{2}$. For each $k=0,1,2, \ldots$ let $f_{k}:[k \pi,(k+1) \pi] \rightarrow[0,1]$ be a linear map with $f_{k}(k \pi)=0$ and $f_{k}((k+1) \pi)=1$. Then define $A(r, x)=x^{\exp (r)}$ for all $r \in R$ and all $x \in[0,1]$. Then $A: R \times[0,1] \rightarrow[0,1]$ is a topological group action of the additive reals on $[0,1]$, that is, a flow. The associated map $\hat{A}: R \rightarrow G([0,1])$ is an imbedding. Also for a fixed $t \in(0,1)$, the map $r \rightarrow t^{\exp (r)}$ is a homeomorphism of $R$ onto $(0,1)$. Now let $c_{0}=\left\{\left(r_{i}\right): r_{i} \in R\right.$ for all $i$ and $\left.r_{i} \rightarrow 0\right\}$. We give $c_{0}$ the supremum norm. Then $c_{0}$ is a separable infinite-dimensional Banach space and hence homeomorphic to $l_{2}$. For each $\left(r_{i}\right) \in c_{0}$ define the function $L\left(\left(r_{i}\right)\right)$ on $X$ by $L\left(\left(r_{i}\right)\right)(x)=f_{k}^{-1}\left(A\left(r_{k}, f_{k}(x)\right)\right)$ for $x \in[k \pi,(k+1) \pi]$ and $L\left(\left(r_{i}\right)\right)(\bar{\alpha})=\bar{\alpha}$ for $\alpha \in[-1,1]$. Then one can show that $L: c_{0} \rightarrow G(X)$ is an imbedding of $c_{0}$ into $G(X)$ as a topological group. The details are fairly routine and will be omitted. Note that $L\left[c_{0}\right] \subset O$.

Now suppose that $f \in O$. Then let $F(f)=\left(r_{i}\right)$ where $r_{k}$ is defined by $f_{k}(f(k \pi+\pi / 2))$ $=(1 / 2)^{\exp \left(r_{k}\right)}$. Then one can show that $F: O \rightarrow c_{0}$ is continuous. Also $F \circ L: c_{0} \rightarrow c_{0}$ is the identity map. Now let $f \in O$ and associate with $f$ the map $Q(f)$ $=(L \circ F(f))^{-1} \circ f$. Then $Q(f) \in G(X)$ and $Q(f)$ leaves the points $k \pi+\pi / 2$ fixed for all $k=0,1,2, \ldots$. The map $Q$ is a continuous retraction of $O$ onto the set of all functions which leave the points $\{k \pi+\pi / 2: k=0,1,2, \ldots\}$ fixed. Now let $P: O \rightarrow c_{0} \times Q[O]$ be defined by $P(f)=(F(f), Q(f))$. Then $P$ is a homeomorphism. 
The inverse of $P$ is just the map $P^{-1}\left(\left(x_{i}\right), f\right)=L\left(\left(x_{i}\right)\right) \circ f$. By Proposition VI.3, $Q[O]$ is homeomorphic to $l_{2}$. Thus $O$ is homeomorphic to $l_{2} \times l_{2}$. Thus $O$ is homeomorphic to $l_{2}$.

Claim 4. The space ker $h$ is homeomorphic to $l_{2}$.

Proof of Claim 4. We simply use Claim 2 and Claim 3 and the result of Henderson in [17]. Any contractible separable Fréchet manifold is homeomorphic to $l_{2}$ and $\operatorname{ker} h$ is a contractible separable Fréchet manifold.

Now clearly $G(X) \sim \operatorname{ker} h \times N$ and thus $G(X) \sim l_{2} \times N$ by Claim 4 . The proof of Theorem VI.8 is now complete.

VI.9. THEOREM. Let $X$ be the sin (1/x)-curve and let $G_{0}(X)$ be the group of homeomorphisms of $X$ which are the identity on the limit arc. Then $G_{0}(X) \sim l_{2} \times N$.

Proof. The topological group homomorphism $h: G(X) \rightarrow N$ defined in the proof of Theorem VI.8 has the property that $h_{0}=h \mid G_{0}(X)$ is onto the even integers. The contraction $H$ of $\operatorname{ker} h$ described in the proof of Claim 2 in the proof of Theorem VI. 8 has the property that it also contracts ker $h_{0}$. Now in Claim 3 in the proof of Theorem VI.8, if we let

$$
O_{0}=O \cap G_{0}(X)=\left\{f \in \operatorname{ker} h_{0}: f(k \pi+\pi / 2) \in(k \pi,(k+1) \pi)\right\},
$$

then $O_{0} \sim c_{0} \times Q\left[O_{0}\right]$ as there. Now appealing to Proposition VI.3 we get that $Q\left[O_{0}\right]$ is homeomorphic to $l_{2}$. Thus $O_{0} \sim l_{2}$ and $\operatorname{ker} h_{0}$ is a separable Fréchet manifold. Thus ker $h_{0} \sim l_{2}$ by [17]. Thus $G_{0}(X) \sim l_{2} \times N$.

VI.10. THEOREM. The group of homeomorphisms of the circle $1 / x$-curve is homeomorphic to $l_{2}$.

Proof. We will use the following model of the circle $1 / x$-curve for this proof. Let $X=R^{+} \cup S^{1}$ where $R^{+}=\{x \in R: x \geqq 0\}$ and $S^{1}=\left\{z \in R^{2}:|z|=1\right\}$. We consider $R^{+}$and $S^{1}$ as disjoint and topologized in the following manner. For $x \in R^{+}$a neighborhood basis for $x$ in $X$ is any neighborhood basis for $x$ in $R^{+}$. Now for $x \in S^{1}, x$ will have as a neighborhood basis neighborhoods of the form $O(r, \varepsilon, x)=\left\{y \in R^{+}: y>r\right.$ and $\left.|\exp (i y)-x|<\varepsilon\right\} \cup\left\{z \in S^{1}:|z-x|<\varepsilon\right\}$ where $r \in R^{+}$and $\varepsilon>0$. In terms of sequences, if $x_{n} \in R^{+}$and $z \in S^{1}$, then $x_{n} \rightarrow z$ in $X$ if and only if $x_{n} \rightarrow \infty$ and $\exp \left(i x_{n}\right) \rightarrow z$.

Now let $h: R \rightarrow R$ be a function. We will say that $h$ is semiperiodic if $h(x+2 \pi)$ $=h(x)+2 \pi$ for all $x \in R$. We now let $f \in G(X)$ and define $F: R \times G(X) \rightarrow R$ by

$$
F(r, f)=\left(\lim _{r \rightarrow \infty}[f(r+2 k \pi)-(r+2 k \pi)]\right)+r .
$$

Claim 1. The map $F$ is continuous and for a fixed $f \in G(X), F(r, f)$ is a semiperiodic homeomorphism of $R$ onto $R$. For a fixed $r \in R, F(r, f)$ is a topological group homomorphism of $G(X)$ onto $R$. Also for a fixed $f \in G(X)$,

$$
\lim _{r \rightarrow \infty}|F(r, f)-f(r)|=0 .
$$


Claim 2. If $h$ is a semiperiodic homeomorphism of $R$ onto $R$, then for any $t \in[0,1], f_{t}(x)=t x+(1-t) h(x)$ is also a semiperiodic homeomorphism of $R$ onto $R$.

Claim 3. The space $G(X)$ is contractible.

Proof of Claim 3. Let $f \in G(X), t \in[0,1]$, and $r \in R^{+}$. Then define $H(t, f)(r)$ $=t r+(1-t) f(r)$. For $z \in S^{1}$, let $\exp (i x)=z$ for $x \in R$ and define $H(t, f)(z)$ $=\exp (i(t x+(1-t) F(x, f)))$. Then $H(t, f) \in G(X)$ and is well defined and $H:[0,1] \times G(X) \rightarrow G(X)$ is a contraction of $G(X)$ to $e$.

Claim 4. The space $G(X)$ is a separable Fréchet manifold.

Proof of Claim 4. Let $O=\{f \in G(X): f(2 k \pi) \in(2 k \pi-\pi, 2 k \pi+\pi)$ for $k=1,2, \ldots$ and $f(1) \neq-1$ in $\left.S^{1}\right\}$. Then $O$ is open in $G(X)$. We will show that $O \sim l_{2}$. Let $f_{k}:[2 k \pi-\pi, 2 k \pi+\pi] \rightarrow[0,1]$ be linear for $k=1,2, \ldots$ with $f_{k}(2 k \pi-\pi)=0$ and $f(2 k \pi+\pi)=1$. Let $A: R \times[0,1] \rightarrow[0,1]$ be defined by $A(r, t)=t^{\exp (r)}$. Then $A$ is a flow on $[0,1]$. Now let $c=\left\{\left(x_{i}\right): x_{i} \in R\right.$ for all $i$ and $x_{i} \rightarrow x_{0}$ for some $\left.x_{0} \in R\right\}$. If we give $c$ the supremum norm, then $c$ becomes a Banach space which is separable and infinite dimensional. Now let $\left(x_{i}\right) \in c$ and suppose that $x_{i} \rightarrow x_{0}$. We define $L: c \rightarrow G(X)$ by $L\left(\left(x_{i}\right)\right)=f$ where $f(x)=x$ for $x \in[0, \pi]$ and $f(x)=f_{k}^{-1} A\left(x_{k}, f_{k}(x)\right)$ for $x \in[2 k \pi-\pi, 2 k \pi+\pi]$, some $k=1,2, \ldots$ Now for $z \in S^{1}$ we define $f(z)$ in the following manner. If $z=-1$, then $f(z)=z$. If $z \neq-1$, then suppose that $\exp (i y)=z$ where $\pi<y<3 \pi$ and define

$$
f(z)=\exp \left(i f_{1}^{-1}\left(A\left(x_{0}, f_{1}(y)\right)\right)\right) \text {. }
$$

Then using the fact that $x_{i} \rightarrow x_{0}$ one can verify that $f$ is a homeomorphism of $X$ onto $X$. The map $L: c \rightarrow G(X)$ is a topological group imbedding. Also $L[c] \subset O$. Now let $f \in O$ and define the map $F: O \rightarrow c$ by $F(f)=\left(x_{i}\right)$ where

$$
f(2 k \pi)=f_{k}^{-1}\left(A\left(x_{k}, f_{k}(2 k \pi)\right)\right) \text {. }
$$

Then $\left(x_{i}\right) \in c$ since $x_{i} \rightarrow x_{0}$, where

$$
f(1)=\exp \left(i f_{1}^{-1}\left(A\left(x_{0}, f_{1}(2 \pi)\right)\right)\right) \text {. }
$$

Now the map $F: O \rightarrow c$ is continuous. Now define $Q: O \rightarrow O$ by $Q(f)$ $=L \circ F(f)^{-1} \circ f$. Then $Q$ is continuous and $Q[O]$ is the set of all $f \in G(X)$ such that $f(1)=1$ in $S^{1}$ and $f(2 k \pi)=2 k \pi$ for $k=0,1,2, \ldots$ Now by Proposition VI. 5 we have that $Q[O] \sim l_{2}$. Define $P: O \rightarrow c \times Q[O]$ by $P(f)=(F(f), Q(f))$. Then $P$ is a homeomorphism. Now $c \sim l_{2}$ and $Q[O] \sim l_{2}$ and thus $O \sim l_{2}$. Since $G(X)$ is a topological group we have shown that $G(X)$ is a separable Fréchet manifold and the proof of Claim 4 is complete.

Now we have that $G(X)$ is a contractible separable Fréchet manifold. Therefore $G(X) \sim l_{2}$ by [17]. The proof of Theorem VI.10 is now complete.

VI.11. THEOREM. Let $X$ be the circle $1 / x$-curve. Let $G_{0}(X)$ be the group of homeomorphisms of $X$ which are the identity on the limit circle. Then $G_{0}(X) \sim l_{2} \times N$.

Proof. We define $h: G_{0}(X) \rightarrow N$ by $h(f)=(1 / 2 \pi) \lim _{k \rightarrow \infty}[f(2 k \pi)-2 k \pi]$. Then $h$ is a topological group homomorphism and is onto the integers $N$. Now ker $h$ is 
contractible by the contraction described in Claim 3 in the proof of Theorem VI.10. Now letting $O_{0}=O \cap G_{0}(X)$ we have that if $L$ is as in the proof of Claim 4 in the proof of Theorem VI.10, $L: c \rightarrow O$, then $L\left[c_{0}\right]=L[c] \cap O_{0}$. Also $F: O \rightarrow c$ has the property that $F\left[O_{0}\right]=c_{0}$. Now $Q \mid O_{0}$ takes $O_{0}$ into $O_{0}$. Defining $P: O_{0} \rightarrow c_{0} \times Q\left[O_{0}\right]$ as $P \mid O_{0}$, then $P$ is a homeomorphism. Now $c_{0}$ is a Banach space and $Q\left[O_{0}\right] \sim l_{2}$ by Proposition VI.5. Thus $O_{0} \sim l_{2}$ and thus ker $h$ is a separable Fréchet manifold. This implies that ker $h \sim l_{2}$ and that $G_{0}(X) \sim l_{2} \times N$.

It should be clear that our techniques could analyze the topological structure of many other $G(X)$ where $X$ is compact or locally compact and has a dense open subspace homeomorphic to either $R^{+}$or $R$. For many spaces of this type $G(X)$ can be analyzed by simply applying the theorems in this section. For others one would have to repeat some of the techniques in order to make the analysis.

\section{REFERENCES}

1. R. D. Anderson, Topological properties of the Hilbert cube and the infinite product of open intervals, Trans. Amer. Math. Soc. 126 (1967), 200-216. MR 34 \#5045.

2. - Spaces of homeomorphisms of finite graphs (preprint).

3. R. D. Anderson and R. H. Bing, A complete elementary proof that Hilbert space is homeomorphic to the countable infinite product of lines, Bull. Amer. Math. Soc. 74 (1968), 771-792. MR 37 \#5847.

4. R. Arens, Topologies for homeomorphism groups, Amer. J. Math. 68 (1946), 593-610. MR 8, 479.

5. A. Beck, On invariant sets, Ann. of Math. (2) 67 (1958), 99-103. MR 19, 1064.

6. C. Bessaga and A. Pełczyński, The space of Lebesgue measurable functions on the interval $[0,1]$ is homeomorphic to the countable infinite product of lines (preprint).

7. B. L. Brechner, On the dimensions of certain spaces of homeomorphisms, Trans. Amer. Math. Soc. 121 (1966), 516-548. MR 32 \#4662.

8. - Topological groups which are not full homeomorphism groups (preprint).

9. A. V. Cernavskiĩ, Local contractibility of the homeomorphism group of a manifold, Dokl. Akad. Nauk SSSR 182 (1968), 510-513=Soviet Math. Dokl. 9 (1968), 1171-1174. MR 38 \#5241.

10. - Local contractability of the group of homeomorphisms of a manifold, Mat. Sb. 79 (121) (1969), 307-356= Math. USSR Sb. 8 (1969), 287-333.

11. W. H. Cutler, Negligible subsets of infinite-dimensional Fréchet manifolds, Proc. Amer. Math. Soc. 23 (1969), 668-675. MR 40 \#2133.

12. J. Dugundji, Topology, Allyn and Bacon, Boston, Mass., 1966. MR 33 \#1824.

13. R. D. Edwards and R. C. Kirby, Deformations of spaces of imbeddings, Ann. of Math. (2) 91 (1971), 63-88.

14. M. K. Fort, Homogeneity of infinite products of manifolds with boundary, Pacific J. Math. 12 (1962), 879-884. MR 26 \#3030.

15. R. Geoghegan, On spaces of homeomorphisms, embeddings and functions (preprint).

16. A. M. Gleason and R. S. Palais, On a class of transformation groups, Amer. J. Math. 79 (1957), 631-648. MR 19, 663.

17. D. W. Henderson, Infinite-dimensional manifolds are open subsets of Hilbert space, Topology 9 (1970), 25-33.

18. W. Hurewicz and H. Wallman, Dimension theory, Princeton Math. Series, vol. 4, Princeton Univ. Press, Princeton, N. J., 1941. MR 3, 312. 
19. M. I. Kadec, Topological equivalence of separable Banach spaces, Dokl. Akad. Nauk SSSR 167 (1966), 23-25= Soviet Math. Dokl. 7 (1966), 319-322. MR 34 \#1828.

20. - A proof of the topological equivalence of all separable infinite-dimensional Banach spaces, Funkcional Anal. i Priložen. 1 (1967), 61-70= Functional Anal. Appl. 1 (1967), 53-62. MR 35 \#700.

21. J. Keesling, Locally compact full homeomorphism groups are zero-dimensional, Proc. Amer. Math. Soc. 29 (1971), 390-396.

22. R. C. Kirby, Stable homeomorphisms and the annulus conjecture, Ann. of Math. (2) 89 (1969), 575-582. MR 39 \#3499.

23. W. K. Mason, The space $H(M)$ of homeomorphisms of a compact manifold onto itself is homeomorphic to $H(M)$ minus any $\sigma$-compact set, Amer. J. Math. 92 (1970), 541-551.

24. E. A. Michael, Topologies on spaces of subsets, Trans. Amer. Math. Soc. 71 (1951), 152-182. MR 13, 54.

25. M. Morse, A special parameterization of curves, Bull. Amer. Math. Soc. 42 (1936), 915-922.

26. D. Montgomery and L. Zippin, Topological transformation groups, Interscience, New York, 1955. MR 17, 383.

27. J. Nagata, Modern dimension theory, Bibliotheca Math., vol. 6, Interscience, New York, 1965. MR $34 \# 8380$.

28. P. L. Renz, The contractibility of the homeomorphism group of some product spaces by Wong's method, Math. Scand. (to appear).

29. H. Whitney, Regular families of curves, Ann. of Math. 34 (1933), 244-270.

30. J. V. Whittaker, On isomorphic groups and homeomorphic spaces, Ann. of Math. (2) 78 (1963), 74-91. MR 27 \#737.

31. R. Y. T. Wong, On homeomorphisms of certain infinite dimensional spaces, Trans. Amer. Math. Soc. 128 (1967), 148-154. MR 35 \#4892.

University of Florida, Gainesville, Florida 32601 